\title{
Capelli Identities for Classical Lie Algebras
}

\author{
Alexander Molev ${ }^{1}$, Maxim Nazarov $^{2}$ \\ ${ }^{1}$ School of Mathematics and Statistics, University of Sydney, \\ Sydney NSW 2006, Australia. E-mail: alexm@maths.usyd.edu.au \\ ${ }^{2}$ Department of Mathematics, University of York, York YO1 5DD, England. \\ E-mail: mln1@york.ac.uk
}

\begin{abstract}
We extend the Capelli identity from the Lie algebra $\mathfrak{g l}_{N}$ to the other classical Lie algebras $\mathfrak{s o}_{N}$ and $\mathfrak{s p}_{N}$. We employ the theory of reductive dual pairs due to Howe.
\end{abstract}

\section{Introduction}

The Capelli identity is one of the most renowned results in classical invariant theory. It gives a set of $N$ distinguished generators for the centre of enveloping algebra $\mathrm{U}\left(\mathfrak{g l}_{N}\right)$ of the general linear Lie algebra. For any positive integer $m$, consider the natural action of the complex Lie group $G L_{N}$ in the ring $\mathcal{P}$ of polynomials on the vector space $\mathbb{C}^{m} \otimes \mathbb{C}^{N}$. The resulting representation $\mathrm{U}\left(\mathfrak{g l}_{N}\right) \rightarrow \mathcal{P} \mathcal{D}$ by differential operators on $\mathbb{C}^{m} \otimes \mathbb{C}^{N}$ with polynomial coefficients is faithful when $m \geqslant N$.

The image of the centre $\mathrm{Z}\left(\mathfrak{g l}_{N}\right)$ of the algebra $\mathrm{U}\left(\mathfrak{g l}_{N}\right)$ under this representation is the subalgebra $\mathcal{I} \subset \mathcal{P D}$ of $G L_{m} \times G L_{N}$-invariant differential operators. Denote $l=\min (m, N)$. The algebra $\mathcal{I}$ has a distinguished set of generators $\Omega_{1}, \ldots, \Omega_{l}$ which are called the Cayley operators $[\mathrm{W}]$. Let $x_{a i}$ with $a=1, \ldots, m$ and $i=1, \ldots, N$ be coordinates on $\mathbb{C}^{m} \otimes \mathbb{C}^{N}$. Let $\partial_{a i}$ be the corresponding partial differentiations. Then $\Omega_{k} \in \mathcal{I}$ is defined for any index $k=1,2, \ldots$ as

$$
\frac{1}{k !} \sum_{\sigma \in S_{k}} \sum_{a_{1}, \ldots, a_{k}} \sum_{i_{1}, \ldots, i_{k}} \operatorname{sgn}(\sigma) \cdot x_{a_{1} i_{1}} \ldots x_{a_{k} i_{k}} \partial_{a_{1} i_{\sigma(1)}} \ldots \partial_{a_{k} i_{\sigma(k)}}
$$

where $\operatorname{sgn}(\sigma)$ is the sign of the element $\sigma$ of the symmetric group $S_{k}$. Here the indices $a_{1}, \ldots, a_{k}$ and $i_{1}, \ldots, i_{k}$ run through $1, \ldots, m$ and $1, \ldots, N$ respectively. The differential operator (1.1) can be rewritten as

$$
\sum_{a_{1}<\ldots<a_{k}} \sum_{i_{1}<\ldots<i_{k}} \operatorname{det}\left[x_{a_{p} i_{q}}\right] \operatorname{det}\left[\partial_{a_{p} i_{q}}\right]
$$

where the determinants are taken with respect to the indices $p, q=1, \ldots, k$. In particular, we have $\Omega_{k}=0$ for any $k>l$.

The Capelli identity [C] gives an explicit formula for a preimage in $\mathrm{Z}\left(\mathfrak{g l}_{N}\right)$ of the Cayley operator $\Omega_{k} \in \mathcal{I}$. Let $E_{i j}$ be the generators of the enveloping algebra $\mathrm{U}\left(\mathfrak{g l}_{N}\right)$ such that in the above representation $\mathrm{U}\left(\mathfrak{g l}_{N}\right) \rightarrow \mathcal{P} \mathcal{D}$

$$
E_{i j} \mapsto \sum_{a} x_{a i} \partial_{a j}
$$


Then $\Omega_{k}$ is the image of the element from $\mathrm{U}\left(\mathfrak{g l}_{N}\right)$ given by

$$
\frac{1}{k !} \sum_{\sigma \in S_{k}} \sum_{i_{1}, \ldots, i_{k}} \operatorname{sgn}(\sigma) \cdot \prod_{s}\left(E_{i_{s} i_{\sigma(s)}}+(s-1) \cdot \delta_{i_{s} i_{\sigma(s)}}\right)
$$

where the index $s$ runs through $1, \ldots, k$ and the factors corresponding to $s$ are arranged from left to right. We write $\delta_{i j}$ for the Kronecker delta. Here (1.3) is the $k$-th Capelli element of the centre $\mathrm{Z}\left(\mathfrak{g l}_{N}\right)$; see $[\mathrm{H}, \mathrm{HU}]$ for further comments.

The algebra $\mathcal{I}$ has another distinguished set of generators $\Theta_{1}, \ldots, \Theta_{l}$. Invariant differential operator $\Theta_{k}$ is defined for any $k=1,2, \ldots$ as

$$
\frac{1}{k !} \sum_{\sigma \in S_{k}} \sum_{a_{1}, \ldots, a_{k}} \sum_{i_{1}, \ldots, i_{k}} x_{a_{1} i_{1}} \ldots x_{a_{k} i_{k}} \partial_{a_{1} i_{\sigma(1)}} \ldots \partial_{a_{k} i_{\sigma(k)}} .
$$

Let $d_{1}, \ldots, d_{m}$ be the multiplicities of the numbers $1, \ldots, m$ respectively in the sequence $\left(a_{1}, \ldots, a_{k}\right)$. Further, let $f_{1}, \ldots, f_{N}$ be the multiplicities of the numbers $1, \ldots, N$ in the sequence $\left(i_{1}, \ldots, i_{k}\right)$. Then the differential operator (1.4) can be rewritten as

$$
\sum_{a_{1} \leqslant \ldots \leqslant a_{k}} \sum_{i_{1} \leqslant \ldots \leqslant i_{k}} \frac{\operatorname{per}\left[x_{a_{p} i_{q}}\right] \operatorname{per}\left[\partial_{a_{p} i_{q}}\right]}{d_{1} ! \ldots d_{m} ! f_{1} ! \ldots f_{N} !}
$$

where

$$
\operatorname{per}\left[x_{a_{p} i_{q}}\right]=\sum_{\sigma \in S_{k}} x_{a_{1} i_{\sigma(1)}} \ldots x_{a_{k} i_{\sigma(k)}}
$$

is the permanent of the matrix $\left[x_{a_{p} i_{q}}\right]$ with the indices $p, q=1, \ldots, k$.

An explicit formula for a preimage in $\mathrm{Z}\left(\mathfrak{g l}_{N}\right)$ of the operator $\Theta_{k}$ has been found by the second author [N1]. Similarly to (1.3), the differential operator $\Theta_{k}$ is the image of the element from $\mathrm{U}\left(\mathfrak{g l}_{N}\right)$

$$
\frac{1}{k !} \sum_{\sigma \in S_{k}} \sum_{i_{1}, \ldots, i_{k}} \prod_{s}\left(E_{i_{s} i_{\sigma(s)}}-(s-1) \cdot \delta_{i_{s} i_{\sigma(s)}}\right)
$$

where the factors corresponding to the index $s=1, \ldots, k$ are again arranged from left to right. One can check that the element (1.5) belongs to $\mathrm{Z}\left(\mathfrak{g l}_{N}\right)$; cf. [N1]. See also the remarks subsequent to the proof of Proposition 3.9 in the present article.

Take an irreducible polynomial $\mathfrak{g l}_{N}$-module of highest weight $\nu=\left(\nu_{1}, \ldots, \nu_{N}\right)$. We make the standard choice of a Borel subalgebra in $\mathfrak{g l}_{N}$; see Section 6. Firstly, observe that the eigenvalue of (1.3) in this module is the elementary symmetric polynomial

$$
e_{k}\left(\nu_{1}, \ldots, \nu_{N}\right)=\sum_{i_{1}<\ldots<i_{k}} \nu_{i_{1}} \ldots \nu_{i_{k}}
$$

plus lower terms. Eigenvalue of (1.5) is up to lower terms the complete symmetric polynomial

$$
h_{k}\left(\nu_{1}, \ldots, \nu_{N}\right)=\sum_{i_{1} \leqslant \ldots \leqslant i_{k}} \nu_{i_{1}} \ldots \nu_{i_{k}} .
$$

Secondly, by the definitions (1.1) and (1.4) the eigenvalues of (1.3) and (1.5) in that module vanish if $\nu_{1}+\ldots+\nu_{N}<k$. These two properties characterize each of the central elements (1.3) and (1.5) of $\mathrm{U}\left(\mathfrak{g l}_{N}\right)$ uniquely; cf. [K1, K2, OO1, S]. 
Now let $\mathfrak{g}$ be the classical Lie algebra $\mathfrak{s o}_{N}$ or $\mathfrak{s p}_{N}$ over $\mathbb{C}$. The rank of the reductive Lie algebra $\mathfrak{g}$ is $n=[N / 2]$. Let $G$ be the corresponding classical Lie group $O_{N}$ or $S p_{N}$ over $\mathbb{C}$. We will denote by $\mathrm{Z}(\mathfrak{g})$ the ring of invariants in the enveloping algebra $\mathrm{U}(\mathfrak{g})$ with respect to the adjoint action of $G$. So the $\operatorname{ring} \mathrm{Z}(\mathfrak{g})$ coincides with the centre of $\mathrm{U}(\mathfrak{g})$ for $\mathfrak{g}=\mathfrak{s o}_{2 n+1}, \mathfrak{s p}_{2 n}$ but is strictly contained in the centre of $\mathrm{U}(\mathfrak{g})$ when $\mathfrak{g}=\mathfrak{s o}_{2 n}$. The principal aim of this article is to construct in $\mathrm{Z}(\mathfrak{g})$ the analogues of the elements $(1.3)$ and (1.5) from $\mathrm{Z}\left(\mathfrak{g l}_{N}\right)$; cf. [OO2].

In Section 2 for each $k=1,2, \ldots$ we define the elements $C_{k}$ and $D_{k}$ of $\mathrm{Z}(\mathfrak{g})$ similarly to the above characterization of the elements (1.3) and (1.5) of $\mathrm{Z}\left(\mathfrak{g l}_{N}\right)$. We will choose a Cartan decomposition $\mathfrak{g}=\mathfrak{n}_{-} \oplus \mathfrak{h} \oplus \mathfrak{n}_{+}$in that section. Now let $\lambda=\left(\lambda_{1}, \ldots, \lambda_{n}\right)$ be a dominant weight of the Cartan subalgebra $\mathfrak{h}$ with integral labels. So $\lambda_{1}, \ldots, \lambda_{n}$ satisfy the inequalities (2.14). Let $V_{\lambda}$ be the irreducible $\mathfrak{g}-$ module of the highest weight $\lambda$. Each of the elements $C_{k}$ and $D_{k}$ of $\mathrm{Z}(\mathfrak{g})$ is defined by the following two conditions; cf. [OO2,S]. First, the eigenvalues of $C_{k}$ and $D_{k}$ in $V_{\lambda}$ are respectively $(-1)^{k} e_{k}\left(\lambda_{1}^{2}, \ldots, \lambda_{n}^{2}\right)$ and $h_{k}\left(\lambda_{1}^{2}, \ldots, \lambda_{n}^{2}\right)$ plus lower terms. Second, both of the elements $C_{k}$ and $D_{k}$ vanish in $V_{\lambda}$ if $\lambda_{1}+\ldots+\lambda_{n-1}+\left|\lambda_{n}\right|<k$. In particular, we have $C_{k}=0$ for $k>n$.

In Section 3 we give explicit formulas for the elements $C_{k}$ and $D_{k}$ in terms of the standard generators of the enveloping algebra $\mathrm{U}(\mathfrak{g})$; see Theorems 3.2 and 3.3 respectively. These formulas are motivated by the results of $[\mathrm{C} 1, \mathrm{~S} 2]$ and $[\mathrm{NO}]$. We also employ the technique developed in our joint article with G. Olshanski [MNO]. In our proofs we follow an approach to the classical Capelli identity from [M2].

We will take the Lie group $G$ as a subgroup in $G L_{N}$. This embedding is also fixed in Section 2. Let us restrict the representation (1.2) to the subalgebra $U(\mathfrak{g})$ in $\mathrm{U}\left(\mathfrak{g l}_{N}\right)$. In Section 4 we evaluate the images of the elements $C_{1}, \ldots, C_{n}$ under this representation for $\mathfrak{g}=\mathfrak{s o}_{N}$; see Theorem 4.1. Further, in Section 5 for $\mathfrak{g}=\mathfrak{s p}_{N}$ we evaluate the images under this representation of the elements $D_{1}, D_{2}, \ldots$; see Theorem 5.1. We regard these two results as generalizations of the Capelli identity from $\mathfrak{g l}_{N}$ to the Lie algebras $\mathfrak{s o}_{N}$ and $\mathfrak{s p}_{N}$ respectively. A different generalization of the Capelli identity has been given in [KS1, KS2].

The algebra of invariants in $\mathcal{P} \mathcal{D}$ with respect to the natural action of the group $G L_{N}$ is generated by the action of the Lie algebra $\mathfrak{g l}_{m}$ in $\mathcal{P}$. Consider the algebra of invariants in $\mathcal{P D}$ with respect to the action of the subgroup $O_{N} \subset G L_{N}$. This algebra is generated $[\mathrm{H}]$ by an action in $\mathcal{P}$ of the Lie algebra $\mathfrak{s p}_{M}$ with $M=2 \mathrm{~m}$. The images of $\mathrm{Z}\left(\mathfrak{s o}_{N}\right)$ and $\mathrm{Z}\left(\mathfrak{s p}_{M}\right)$ in $\mathcal{P} \mathcal{D}$ coincide. For each $k=1, \ldots, m$ we express the image of $C_{k} \in \mathrm{Z}\left(\mathfrak{s p}_{M}\right)$ in $\mathcal{P D}$ as a linear combination of the images of $C_{1}, \ldots, C_{k} \in \mathrm{Z}\left(\mathfrak{s o}_{N}\right)$; see Theorem 4.4. This result is based on the fact that the real Lie groups $O_{N}(\mathbb{R})$ and $S p_{M}(\mathbb{R})$ form a reductive dual pair inside the group $S p_{M N}(\mathbb{R})$; cf. [HU,KV]. Along with Theorem 4.1 this result provides an explicit formula for the image of $C_{k} \in \mathrm{Z}\left(\mathfrak{s p}_{M}\right)$ in $\mathcal{P D}$.

Now consider the algebra of invariants in $\mathcal{P} \mathcal{D}$ under the natural action of the subgroup $S p_{N} \subset G L_{N}$. This algebra is generated by an action in $\mathcal{P}$ of the Lie algebra $\mathfrak{s o}_{M}$. The images of $\mathrm{Z}\left(\mathfrak{s p}_{N}\right)$ and $\mathrm{Z}\left(\mathfrak{s o}_{M}\right)$ in $\mathcal{P D}$ again coincide. For each $k=1,2, \ldots$ we express the image of $D_{k} \in \mathrm{Z}\left(\mathfrak{s o}_{M}\right)$ in $\mathcal{P} \mathcal{D}$ as a linear combination of the images of $D_{1}, \ldots, D_{k} \in \mathrm{Z}\left(\mathfrak{s p}_{N}\right)$; see Theorem 5.3. It is based on the fact that the Lie groups $S p_{N}(\mathbb{R})$ and $O_{M}^{*}(\mathbb{R})$ also form a reductive dual pair. Along with Theorem 5.1 it provides explicit formula for the image of $D_{k} \in \mathrm{Z}\left(\mathfrak{s p}_{M}\right)$ in $\mathcal{P} \mathcal{D}$.

In Section 6 we consider a certain generating function $C(u)$ for the elements $C_{1}, \ldots, C_{n} \in \mathrm{Z}(\mathfrak{g})$. This function is defined by (4.8) for $\mathfrak{g}=\mathfrak{s o}_{N}$ and by (5.10) 
for $\mathfrak{g}=\mathfrak{s p}_{N}$. The main result of this section is Theorem 6.2. It gives an explicit formula for the function $C(u)$ in terms of the standard generators of the algebra $\mathrm{U}(\mathfrak{g})$. Moreover, $C(u)$ is invertible as a formal power series in $u^{-1}$ and the inverse series can be regarded as a generating function for the elements $D_{1}, D_{2}, \ldots \in \mathrm{Z}(\mathfrak{g})$; see (4.12) if $\mathfrak{g}=\mathfrak{s o}_{N}$ and (5.11) if $\mathfrak{g}=\mathfrak{s p}_{N}$. Theorem 6.2 was obtained in 1991 as a continuation of $[\mathrm{O}]$ and served as a starting point for the present work.

A $q$-analogue of the classical Capelli identity has been given in [NUW1]. In the particular $m=1$ case $q$-analogues of our Theorems 4.1 and 4.4 have been obtained in [NUW2]. It would be interesting to find $q$-analogues of all our results.

We are grateful to A. Okounkov and G. Olshanski for kindly informing us about their results on the factorial Schur functions [OO1] at an early stage of our work. We are also grateful to R. Howe, P. Jarvis, T. Koornwinder, L. Kovács, A. Lascoux, B. Leclerc, S. Okada, C. Procesi and J.-Y. Thibon for helpful discussions and valuable remarks. The second author has been supported by an EPSRC Advanced Research Fellowship and by EC TMR grant FMRX-CT97-0100.

\section{Two families of generators in $\mathrm{Z}(\mathfrak{g})$}

In this section we will introduce two distinguished families of generators in the ring $\mathrm{Z}(\mathfrak{g})$ of $G$-invariants in the enveloping algebra $U(\mathfrak{g})$. Consider the vector space of symmetric polynomials in the independent variables $z_{1}, \ldots, z_{n}$ with complex coefficients where $n$ is the rank of the simple Lie algebra $\mathfrak{g}$. We will make use of a special basis in this vector space consisting of factorial Schur polynomials. Let us start with recalling several facts about these polynomials from [BL, M, OO1].

Fix an arbitrary sequence of complex numbers $a=\left(a_{1}, a_{2}, \ldots\right)$. Then for each $k=0,1,2, \ldots$ introduce the $k$-th generalized factorial power of a variable $z$

$$
(z \mid a)^{k}=\left(z-a_{1}\right) \cdots\left(z-a_{k}\right)
$$

Let $\mu=\left(\mu_{1}, \mu_{2}, \ldots\right)$ be a partition with length $\ell(\mu) \leqslant n$. Consider the function

$$
s_{\mu}\left(z_{1}, \ldots, z_{n} \mid a\right)=\frac{\operatorname{det}\left[\left(z_{q} \mid a\right)^{\mu_{p}+n-p}\right]}{\operatorname{det}\left[\left(z_{q} \mid a\right)^{n-p}\right]}
$$

where the determinants are taken with respect to the indices $p, q=1, \ldots, n$. This function is a symmetric polynomial in $z_{1}, \ldots, z_{n}$ which is called the generalized factorial Schur polynomial [M,Example I.3.20]. Note that here the denominator

$$
\operatorname{det}\left[\left(z_{q} \mid a\right)^{n-p}\right]=\prod_{p<q}\left(z_{p}-z_{q}\right)
$$

is the Vandermonde determinant. Thus it does not depend on the sequence $a$.

If $a=(0,0, \ldots)$ the function $s_{\mu}\left(z_{1}, \ldots, z_{n} \mid a\right)$ is the ordinary Schur polynomial $s_{\mu}\left(z_{1}, \ldots, z_{n}\right)$. For the general sequence $a$ the definition (2.1) implies that

$$
s_{\mu}\left(z_{1}, \ldots, z_{n} \mid a\right)=s_{\mu}\left(z_{1}, \ldots, z_{n}\right)+\text { lower degree terms. }
$$

Hence the polynomials $s_{\mu}\left(z_{1}, \ldots, z_{n} \mid a\right)$ with $\ell(\mu) \leqslant n$ form a linear basis in the ring of symmetric polynomials in the variables $z_{1}, \ldots, z_{n}$. 
From now on we will suppose that the sequence $a$ is multiplicity-free, that is $a_{k} \neq a_{l}$ for all $k \neq l$. We will use the following characterisation of the polynomial $s_{\mu}\left(z_{1}, \ldots, z_{n} \mid a\right)$; cf. [OO1, Theorem 3.3]. For any partition $\lambda=\left(\lambda_{1}, \lambda_{2}, \ldots\right)$ such that $l(\lambda) \leqslant n$ introduce the following $n$-tuple of elements from $a$ :

$$
a_{\lambda}=\left(a_{\lambda_{1}+n}, \ldots, a_{\lambda_{n}+1}\right)
$$

As usual, we will write $|\lambda|$ for the degree $\lambda_{1}+\lambda_{2}+\ldots$ of the partition $\lambda$.

Theorem 2.1. Let $f\left(z_{1}, \ldots, z_{n}\right)$ be a symmetric polynomial of degree not more than $|\mu|$. Then the following three conditions are equivalent:

i) $f\left(z_{1}, \ldots, z_{n}\right)$ equals $s_{\mu}\left(z_{1}, \ldots, z_{n} \mid a\right)$ up to a scalar multiple;

ii) $f\left(a_{\lambda}\right)=0$ for any $\lambda$ such that $\lambda_{k}<\mu_{k}$ for at least one index $k$;

iii) $f\left(a_{\lambda}\right)=0$ for any $\lambda$ such that $|\lambda|<|\mu|$ while the leading component of the polynomial $f\left(z_{1}, \ldots, z_{n}\right)$ is $s_{\mu}\left(z_{1}, \ldots, z_{n}\right)$ up to a scalar multiple.

Proof. Let us check first that the polynomial $s_{\mu}\left(z_{1}, \ldots, z_{n} \mid a\right)$ satisfies (ii). For $\left(z_{1}, \ldots, z_{n}\right)=a_{\lambda}$ the $(p, q)$-entry of the matrix in the numerator in (2.1) is

$$
\left(a_{\lambda_{q}+n-q+1}-a_{1}\right) \ldots\left(a_{\lambda_{q}+n-q+1}-a_{\mu_{p}+n-p}\right) .
$$

Suppose that $\lambda_{k}<\mu_{k}$ for some index $k$. Then for $p \leqslant k \leqslant q$ we have

$$
1 \leqslant \lambda_{q}+n-q+1 \leqslant \lambda_{k}+n-k+1 \leqslant \mu_{k}+n-k \leqslant \mu_{p}+n-p .
$$

Therefore every entry (2.4) with $p \leqslant k \leqslant q$ is zero. So the numerator in (2.1) vanishes at $\left(z_{1}, \ldots, z_{n}\right)=a_{\lambda}$. The denominator (2.2) does not vanish at $a_{\lambda}$ since the sequence $a$ is multiplicity-free. Hence $s_{\mu}\left(a_{\lambda} \mid a\right)=0$.

Let us also show that $s_{\mu}\left(a_{\mu} \mid a\right) \neq 0$. Indeed, for $\lambda=\mu$ the $(p, q)$-entry $(2.4)$ equals

$$
\left(a_{\mu_{q}+n-q+1}-a_{1}\right) \ldots\left(a_{\mu_{q}+n-q+1}-a_{\mu_{p}+n-p}\right)
$$

which is zero for $p<q$ and non-zero for $p=q$. So the square matrix in the numerator of $(2.1)$ with $\left(z_{1}, \ldots, z_{n}\right)=a_{\mu}$ is lower-triangular with non-zero diagonal entries. So $s_{\mu}\left(a_{\mu} \mid a\right) \neq 0$.

Note that the polynomial $s_{\mu}\left(z_{1}, \ldots, z_{n} \mid a\right)$ satisfies the condition (iii); see (2.3).

Let us now fix any symmetric polynomial $f\left(z_{1}, \ldots, z_{n}\right)$ of degree not more than $|\mu|$. Suppose that the condition (ii) is satisfied. Let us represent this polynomial as a linear combination of the polynomials $s_{\nu}\left(z_{1}, \ldots, z_{n} \mid a\right)$ :

$$
f(x)=c_{\mu} s_{\mu}\left(z_{1}, \ldots, z_{n} \mid a\right)+\sum_{\nu \neq \mu} c_{\nu} s_{\nu}\left(z_{1}, \ldots, z_{n} \mid a\right) ; \quad c_{\mu}, c_{\nu} \in \mathbb{C}
$$

Here we assume that $|\nu| \leqslant|\mu|$. Note that under this assumption the condition $\nu \neq \mu$ is equivalent to existence of an index $k$ such that $\nu_{k}<\mu_{k}$. Denote by $\mathcal{S}$ the set of all possible partitions $\nu$. Let the partition $\lambda$ also run through the set $\mathcal{S}$. Put $\left(z_{1}, \ldots, z_{n}\right)=a_{\lambda}$ in (2.5). Then we obtain the system of linear equations on the coefficients $c_{\nu}$

$$
\sum_{\nu \in \mathcal{S}} c_{\nu} s_{\nu}\left(a_{\lambda} \mid a\right)=0 ; \quad \lambda \in \mathcal{S}
$$


Equip the set $\mathcal{S}$ with any linear ordering $\prec$ such that $|\lambda|<|\nu|$ implies $\lambda \prec \nu$. By arranging the rows and columns of the matrix $\left[s_{\nu}\left(a_{\lambda} \mid a\right)\right]$ according to the ordering $\prec$ we make this matrix triangular with non-zero diagonal entries. Hence $c_{\nu}=0$ for every $\nu \in \mathcal{S}$. So the polynomial $f\left(z_{1}, \ldots, z_{n}\right)$ satisfies (i) by $(2.5)$. The proof that the condition (iii) on $f\left(z_{1}, \ldots, z_{n}\right)$ implies (i) is quite similar

Let us now define the factorial elementary and complete symmetric polynomials by

$$
\begin{aligned}
e_{k}\left(z_{1}, \ldots, z_{n} \mid a\right) & =s_{\left(1^{k}\right)}\left(z_{1}, \ldots, z_{n} \mid a\right) \\
h_{k}\left(z_{1}, \ldots, z_{n} \mid a\right) & =s_{(k)}\left(z_{1}, \ldots, z_{n} \mid a\right)
\end{aligned}
$$

respectively. We also have the following explicit formulas for these polynomials.

Proposition 2.2. We have the equalities

$$
\begin{aligned}
e_{k}\left(z_{1}, \ldots, z_{n} \mid a\right) & =\sum_{1 \leqslant p_{1}<\ldots<p_{k} \leqslant n}\left(z_{p_{1}}-a_{p_{1}}\right)\left(z_{p_{2}}-a_{p_{2}-1}\right) \cdots\left(z_{p_{k}}-a_{p_{k}-k+1}\right), \\
h_{k}\left(z_{1}, \ldots, z_{n} \mid a\right) & =\sum_{1 \leqslant p_{1} \leqslant \ldots \leqslant p_{k} \leqslant n}\left(z_{p_{1}}-a_{p_{1}}\right)\left(z_{p_{2}}-a_{p_{2}+1}\right) \cdots\left(z_{p_{k}}-a_{p_{k}+k-1}\right) .
\end{aligned}
$$

Proof. We will make use of Theorem 2.1. Denote respectively by $e_{k}^{\prime}\left(z_{1}, \ldots, z_{n} \mid a\right)$ and $h_{k}^{\prime}\left(z_{1}, \ldots, z_{n} \mid a\right)$ the polynomials on the right hand sides of the above relations.

Let us prove first that these polynomials are symmetric in $z_{1}, \ldots, z_{n}$. It suffices to demonstrate that they are invarant under the transposition of $z_{i}$ and $z_{i+1}$ for each $i=1, \ldots, n-1$. But this reduces our task to the case $n=2$. Then we have $e_{k}^{\prime}\left(z_{1}, z_{2} \mid a\right)=0$ for $k>2$ while both $e_{1}^{\prime}\left(z_{1}, z_{2} \mid a\right)$ and $e_{2}^{\prime}\left(z_{1}, z_{2} \mid a\right)$ are obviously symmetric. The symmetry of the polynomial $h_{k}^{\prime}\left(z_{1}, z_{2} \mid a\right)$ follows from the equality

$$
h_{k}^{\prime}\left(z_{1}, z_{2} \mid a\right)=\frac{\left(z_{1} \mid a\right)^{k+1}-\left(z_{2} \mid a\right)^{k+1}}{z_{1}-z_{2}}
$$

which is easy to verify. Indeed, the product $\left(z_{1}-z_{2}\right) \cdot h_{k}^{\prime}\left(z_{1}, z_{2} \mid a\right)$ equals

$$
\begin{array}{r}
\left(z_{1}-z_{2}\right) \cdot \sum_{l=0}^{k}\left(z_{1}-a_{1}\right) \ldots\left(z_{1}-a_{l}\right)\left(z_{2}-a_{l+2}\right) \ldots\left(z_{2}-a_{k+1}\right)= \\
\sum_{l=0}^{k}\left(z_{1}-a_{1}\right) \ldots\left(z_{1}-a_{l+1}\right)\left(z_{2}-a_{l+2}\right) \ldots\left(z_{2}-a_{k+1}\right)- \\
\quad \sum_{l=0}^{k}\left(z_{1}-a_{1}\right) \ldots\left(z_{1}-a_{l}\right)\left(z_{2}-a_{l+1}\right) \ldots\left(z_{2}-a_{k+1}\right) .
\end{array}
$$

All summands at the right hand side of the above equality cancel each other except

$$
\left(z_{1}-a_{1}\right) \ldots\left(z_{1}-a_{k+1}\right)-\left(z_{2}-a_{1}\right) \ldots\left(z_{2}-a_{k+1}\right)=\left(z_{1} \mid a\right)^{k+1}-\left(z_{2} \mid a\right)^{k+1} .
$$

Polynomials $e_{k}\left(z_{1}, \ldots, z_{n} \mid a\right), e_{k}^{\prime}\left(z_{1}, \ldots, z_{n} \mid a\right)$ have the same leading components and so do the polynomials $h_{k}\left(z_{1}, \ldots, z_{n} \mid a\right), h_{k}^{\prime}\left(z_{1}, \ldots, z_{n} \mid a\right)$. Thus to complete the 
proof of Proposition 2.2 it remains to check that $e_{k}^{\prime}\left(a_{\lambda} \mid a\right)=0$ and $h_{k}^{\prime}\left(a_{\lambda} \mid a\right)=0$ for $|\lambda|<k$. By the symmetry of polynomials $e_{k}^{\prime}\left(z_{1}, \ldots, z_{n} \mid a\right)$ and $h_{k}^{\prime}\left(z_{1}, \ldots, z_{n} \mid a\right)$

$$
\begin{aligned}
e_{k}^{\prime}\left(z_{1}, \ldots, z_{n} \mid a\right) & =\sum_{1 \leqslant p_{1}<\ldots<p_{k} \leqslant n}\left(z_{n-p_{1}+1}-a_{p_{1}}\right) \cdots\left(z_{n-p_{k}+1}-a_{p_{k}-k+1}\right), \\
h_{k}^{\prime}\left(z_{1}, \ldots, z_{n} \mid a\right) & =\sum_{1 \leqslant p_{1} \leqslant \ldots \leqslant p_{k} \leqslant n}\left(z_{n-p_{1}+1}-a_{p_{1}}\right) \cdots\left(z_{n-p_{k}+1}-a_{p_{k}+k-1}\right) .
\end{aligned}
$$

Note that $p_{1} \leqslant n-k+1$ in the first of these equalities. Thus if $\lambda_{k}=0$ then $\left(a_{\lambda}\right)_{n-p_{1}+1}=a_{p_{1}}$ which implies that $e_{k}^{\prime}\left(a_{\lambda} \mid a\right)=0$. Finally, consider the last formula for $h_{k}^{\prime}\left(z_{1}, \ldots, z_{n} \mid a\right)$. If $\lambda_{1}<k$ then

$$
0 \leqslant \lambda_{n-p_{1}+1} \leqslant \cdots \leqslant \lambda_{n-p_{k}+1} \leqslant k-1 .
$$

These inequalities imply that there exists $i \in\{1, \ldots, k\}$ such that $\lambda_{n-p_{i}+1}=i-1$. Then $\left(a_{\lambda}\right)_{n-p_{i}+1}=a_{p_{i}+i-1}$ and $h_{k}^{\prime}\left(a_{\lambda} \mid a\right)=0$

In particular, $e_{k}\left(z_{1}, \ldots, z_{n} \mid a\right)=0$ for $k>n$. Consider the rational function in $t$

$$
X(t \mid a)=\frac{\left(t-z_{1}\right) \ldots\left(t-z_{n}\right)}{\left(t-a_{1}\right) \ldots\left(t-a_{n}\right)} .
$$

Next proposition shows that $X(t \mid a)$ and $X(t \mid a)^{-1}$ can be regarded as generating functions for the families of symmetric polynomials (2.6) and (2.7).

Proposition 2.3. We have the equalities of formal power series in $t^{-1}$

$$
\begin{aligned}
& 1+\sum_{k=1}^{n} \frac{(-1)^{k} e_{k}\left(z_{1}, \ldots, z_{n} \mid a\right)}{\left(t-a_{n-k+1}\right) \ldots\left(t-a_{n}\right)}=X(t \mid a), \\
& 1+\sum_{k=1}^{\infty} \frac{h_{k}\left(z_{1}, \ldots, z_{n} \mid a\right)}{\left(t-a_{n+1}\right) \cdots\left(t-a_{n+k}\right)}=X(t \mid a)^{-1} .
\end{aligned}
$$

Proof. We will employ the arguments from [OO1, Theorem 12.1]. Let us prove (2.8) first. There is a unique decomposition

$$
\frac{\left(t-z_{1}\right) \ldots\left(t-z_{n}\right)}{\left(t-a_{1}\right) \ldots\left(t-a_{n}\right)}=1+\sum_{k=1}^{n} \frac{c_{k}\left(z_{1}, \ldots, z_{n}\right)}{\left(t-a_{n-k+1}\right) \ldots\left(t-a_{n}\right)}
$$

where $c_{k}\left(z_{1}, \ldots, z_{n}\right)$ is a certain symmetric polynomial in $z_{1}, \ldots, z_{n}$ of degree $k$. Moreover, the leading component of this polynomial coincides with that of $(-1)^{k} e_{k}\left(z_{1}, \ldots, z_{n} \mid a\right)$. Due to Theorem 2.1 it suffices to prove that $c_{k}\left(a_{\lambda}\right)=0$ for any partition $\lambda$ with $|\lambda|<k$. Let us substitute $\left(z_{1}, \ldots, z_{n}\right)=a_{\lambda}$ with $\ell(\lambda)<k$ in (2.10). At the left hand side we get the function of $t$

$$
\prod_{p=1}^{k-1} \frac{t-a_{\lambda_{p}+n-p+1}}{t-a_{n-p+1}}
$$

This rational function obviously has less than $k$ poles. Therefore the coefficient $c_{k}\left(a_{\lambda}\right)$ at the right hand side vanishes. 
The proof of the identity (2.9) is similar. Consider the unique decomposition

$$
\frac{\left(t-a_{1}\right) \ldots\left(t-a_{n}\right)}{\left(t-z_{1}\right) \ldots\left(t-z_{n}\right)}=1+\sum_{k=1}^{\infty} \frac{d_{k}\left(z_{1}, \ldots, z_{n}\right)}{\left(t-a_{n+1}\right) \cdots\left(t-a_{n+k}\right)}
$$

where $d_{k}\left(z_{1}, \ldots, z_{n}\right)$ is a certain symmetric polynomial in $z_{1}, \ldots, z_{n}$ of degree $k$. The leading component of this polynomial coincides with that of $h_{k}\left(z_{1}, \ldots, z_{n} \mid a\right)$. It remains to prove that $d_{k}\left(a_{\lambda}\right)=0$ for any partition $\lambda$ with $|\lambda|<k$. Let us substitute $\left(z_{1}, \ldots, z_{n}\right)=a_{\lambda}$ in (2.11). At the left hand side we get a rational function in $t$ with simple poles only at $t=a_{\lambda_{p}+n-p+1}$ where $\lambda_{p}+n-p+1>n$, that is where $\lambda_{p} \geqslant p$. The number of such indices $p$ cannot exceed $\lambda_{1}$. So the coefficient $d_{k}(\lambda)$ with $k>\lambda_{1}$ at the right hand side vanishes

We will use the symmetric polynomials (2.6) and (2.7) to define some distinguished elements $C_{k}$ and $D_{k}$ in the ring of invariants $\mathrm{Z}(\mathfrak{g})$. From now on we will let the indices $i, j$ run through the set $\{-n, \ldots,-1,1, \ldots, n\}$ if $N=2 n$ and the set $\{-n, \ldots,-1,0,1, \ldots, n\}$ if $N=2 n+1$. Let $e_{i}$ form the standard basis in $\mathbb{C}^{N}$. We will realize the group $G=O_{N}$ as the subgroup in $G L_{N}$ preserving the symmetric bilinear form $\left\langle e_{i}, e_{j}\right\rangle=\delta_{i,-j}$. The group $G=S p_{N}$ will be realized as the subgroup in $G L_{N}$ preserving the alternating form $\left\langle e_{i}, e_{j}\right\rangle=\delta_{i,-j} \cdot \operatorname{sgn} i$.

Put $\varepsilon_{i j}=\operatorname{sgn} i \cdot \operatorname{sgn} j$ if $G=S p_{N}$ and $\varepsilon_{i j}=1$ if $G=O_{N}$. Let $E_{i j} \in \operatorname{End}\left(\mathbb{C}^{N}\right)$ be the standard matrix units. We will also regard $E_{i j}$ as basis elements of the Lie algebra $\mathfrak{g l}_{N}$. The Lie subalgebra $\mathfrak{g} \in \mathfrak{g l}_{N}$ is then spanned by the elements $F_{i j}=E_{i j}-\varepsilon_{i j} \cdot E_{-j,-i}$. Now let us fix the Cartan decomposition $\mathfrak{g}=\mathfrak{n}_{-} \oplus \mathfrak{h} \oplus \mathfrak{n}_{+}$ as follows. The nilpotent subalgebras $\mathfrak{n}_{+}$and $\mathfrak{n}_{-}$are spanned by the elements $F_{i j}$ where $i<j$ and $i>j$ respectively. The Cartan subalgebra $\mathfrak{h}$ is spanned by the elements $F_{i i}$. Further, we will fix the basis $\left(F_{-n,-n}, \ldots, F_{-1,-1}\right)$ in $\mathfrak{h}$. Any weight $\lambda=\left(\lambda_{1}, \ldots, \lambda_{n}\right)$ of $\mathfrak{h}$ will be taken with respect to this basis. The half-sum of the positive roots of $\mathfrak{h}$ is then

$$
\rho=(\varepsilon+n-1, \varepsilon+n-2, \ldots, \varepsilon)
$$

where $\varepsilon=0, \frac{1}{2}, 1$ for $\mathfrak{g}=\mathfrak{s o}_{2 n}, \mathfrak{s o}_{2 n+1}, \mathfrak{s p}_{2 n}$ respectively. Denote $\lambda_{p}+\rho_{p}=l_{p}$. Recall that the Harish-Chandra homomorphism $\omega: U(\mathfrak{g})^{\mathfrak{h}} \rightarrow \mathrm{U}(\mathfrak{h})$ is defined by

$$
\omega(X)-X \in \mathrm{U}(\mathfrak{g}) \mathfrak{n}_{+} \cap \mathrm{U}(\mathfrak{g})^{\mathfrak{h}}=\mathfrak{n}_{-} \mathrm{U}(\mathfrak{g}) \cap \mathrm{U}(\mathfrak{g})^{\mathfrak{h}}
$$

We will identify the algebra $U(\mathfrak{h})=S(\mathfrak{h})$ with the algebra of polynomial functions on the space $\mathfrak{h}^{*}$. Then the restriction of $\omega$ to the subalgebra $\mathrm{Z}(\mathfrak{g}) \subset U(\mathfrak{g})^{\mathfrak{h}}$ is an isomorphism onto the algebra of symmetric polynomials in the variables $l_{1}^{2}, \ldots, l_{n}^{2}$ [D, Theorem 7.4.5]. We will call this restriction the Harish-Chandra isomorphism.

Let us now make a choice of the sequence $a$ in the definition (2.1). Namely, set

$$
a=\left(\varepsilon^{2},(\varepsilon+1)^{2},(\varepsilon+2)^{2}, \ldots\right) .
$$

Note that here $\left\{a_{1}, \ldots, a_{n}\right\}=\left\{\rho_{1}^{2}, \ldots, \rho_{n}^{2}\right\}$. Then define the elements $C_{k}$ and $D_{k}$ in $\mathrm{Z}(\mathfrak{g})$ as the preimages with respect to the Harish-Chandra isomorphism of the symmetric polynomials $(-1)^{k} e_{k}\left(l_{1}^{2}, \ldots, l_{n}^{2} \mid a\right)$ and $h_{k}\left(l_{1}^{2}, \ldots, l_{n}^{2} \mid a\right)$ respectively. Note that $C_{k}=0$ for any $k>n$. Obviously, we have the following proposition. 
Proposition 2.4. Each of the families $C_{1}, \ldots, C_{n}$ and $D_{1}, \ldots, D_{n}$ generates $\mathrm{Z}(\mathfrak{g})$.

In the next section we will give explicit formulas for the elements $C_{k}, D_{k} \in \mathrm{Z}(\mathfrak{g})$ for any $k$ in terms of the standard generators $F_{i j}$ of the enveloping algebra $\mathrm{U}(\mathfrak{g})$.

We will use a corollary to Theorem 2.1. Let $\lambda=\left(\lambda_{1}, \ldots, \lambda_{n}\right)$ be any dominant weight of $\mathfrak{h}$ with integral labels. So $\lambda_{1}, \ldots, \lambda_{n}$ satisfy the inequalities

$$
\begin{array}{ll}
\lambda_{1} \geqslant \ldots \geqslant \lambda_{n} \geqslant 0 & \text { for } \quad \mathfrak{g}=\mathfrak{s o}_{2 n+1}, \mathfrak{s p}_{2 n} \\
\lambda_{1} \geqslant \ldots \geqslant \lambda_{n-1} \geqslant\left|\lambda_{n}\right| & \text { for } \quad \mathfrak{g}=\mathfrak{s o}_{2 n} .
\end{array}
$$

In the case $\mathfrak{g}=\mathfrak{s o}_{2 n}$ we denote $\lambda^{*}=\left(\lambda_{1}, \ldots, \lambda_{n-1},-\lambda_{n}\right)$. Let $V_{\lambda}$ be the irreducible $\mathfrak{g}$-module of the highest weight $\lambda$. Note that for $\mathfrak{g}=\mathfrak{s o}_{2 n}$ the eigenvalues of any element from $\mathrm{Z}(\mathfrak{g})$ in the irreducible modules $V_{\lambda}$ and $V_{\lambda^{*}}$ coincide. We will assume that $\lambda_{n} \geqslant 0$ always, so that $\lambda$ will be always a partition with $\ell(\lambda) \leqslant n$.

Consider the standard $\mathbb{Z}$-filtration on the enveloping algebra $\mathrm{U}(\mathfrak{g})$. Let $Z$ be any element in the ring $\mathrm{Z}(\mathfrak{g})$ of degree not more than $2 k$. Then here is the corollary.

Corollary 2.5. Suppose that $Z$ vanishes in every module $V_{\lambda}$ with $\ell(\lambda)<k$ or in every module $V_{\lambda}$ with $\lambda_{1}<k$. Then $Z$ equals respectively $C_{k}$ or $D_{k}$ up to a scalar multiple.

Proof. The eigenvalue of the element $Z \in \mathrm{Z}(\mathfrak{g})$ is a certain symmetric polynomial in $l_{1}^{2}, \ldots, l_{n}^{2}$. Let us denote by $f$ this polynomial, its degree does not exceed $k$. By our choice (2.13) of the sequence $a$ we have $\left(l_{1}^{2}, \ldots, l_{n}^{2}\right)=a_{\lambda}$. Thus $f\left(a_{\lambda}\right)=0$ for every $\lambda$ such that $\ell(\lambda)<k$ or $\lambda_{1}<k$. Theorem 2.1 now implies that $f\left(l_{1}^{2}, \ldots, l_{n}^{2}\right)$ equals respectively $e_{k}\left(l_{1}^{2}, \ldots, l_{n}^{2} \mid a\right)$ or $h_{k}\left(l_{1}^{2}, \ldots, l_{n}^{2} \mid a\right)$ up to a scalar multiple for any $\lambda$. But the latter two expressions are the eigenvalues in $V_{\lambda}$ of the elements $(-1)^{k} C_{k}$ and $D_{k}$ respectively. So we get the corollary since any element of $\mathrm{Z}(\mathfrak{g})$ is uniquely determined by its eigenvalues in the irreducible modules $V_{\lambda}$

\section{Explicit formulas for the elements $C_{k}$ and $D_{k}$}

In this section we employ the technique developed in [MNO, NO] and [M1, M2, N2].

As in the previous section, let $E_{i j} \in \operatorname{End}\left(\mathbb{C}^{N}\right)$ be the standard matrix units. But here we will regard the elements $F_{i j}=E_{i j}-\varepsilon_{i j} \cdot E_{-j,-i}$ as generators of the enveloping algebra $\mathrm{U}(\mathfrak{g})$. Let $u$ be a complex variable. Introduce the element

$$
F=\sum_{i j} E_{i j} \otimes F_{j i} \in \operatorname{End}\left(\mathbb{C}^{N}\right) \otimes \mathrm{U}(\mathfrak{g})
$$

and denote

$$
F(u)=F-u-\eta \in \operatorname{End}\left(\mathbb{C}^{N}\right) \otimes \mathrm{U}(\mathfrak{g})[u]
$$

where we set $\eta=\frac{1}{2},-\frac{1}{2}$ for $\mathfrak{g}=\mathfrak{s o}_{N}, \mathfrak{s p}_{N}$ respectively. We will use this notation throughout the present section. Consider the elements of the algebra $\operatorname{End}\left(\mathbb{C}^{N}\right)^{\otimes 2}$

$$
P=\sum_{i j} E_{i j} \otimes E_{j i}, \quad Q=\sum_{i j} \varepsilon_{i j} \cdot E_{i j} \otimes E_{-i,-j}
$$

The element $P$ corresponds to the exchange operator $e_{i} \otimes e_{j} \mapsto e_{j} \otimes e_{i}$ in $\left(\mathbb{C}^{N}\right)^{\otimes 2}$. The element $Q$ is obtained from $P$ by applying to either of the tensor factors in 
End $\left(\mathbb{C}^{N}\right)^{\otimes 2}$ the transposition with respect to the bilinear form $\langle$,$\rangle . Observe that$

$$
P Q=Q P=\left\{\begin{aligned}
Q & \text { if } \mathfrak{g}=\mathfrak{s o}_{N} \\
-Q & \text { if } \mathfrak{g}=\mathfrak{s p}_{N}
\end{aligned}\right.
$$

Denote

$$
R(u, v)=1-\frac{P}{u-v}, \quad \tilde{R}(u, v)=1+\frac{Q}{u+v}
$$

in $\operatorname{End}\left(\mathbb{C}^{N}\right)^{\otimes 2}(u, v)$. The first of these two functions is the rational Yang $R$-matrix.

We will denote by $\iota_{p}$ the embedding of the algebra $\operatorname{End}\left(\mathbb{C}^{N}\right)$ into a finite tensor product End $\left(\mathbb{C}^{N}\right)^{\otimes m}$ as the $p$-th tensor factor:

$$
\iota_{p}(X)=1^{\otimes(p-1)} \otimes X \otimes 1^{\otimes(m-p)} ; \quad p=1, \ldots, m .
$$

Then put

$$
F_{p}(u)=\left(\iota_{p} \otimes \mathrm{id}\right)(F(u)) \in \operatorname{End}\left(\mathbb{C}^{N}\right)^{\otimes m} \otimes \mathrm{U}(\mathfrak{g})[u]
$$

Direct calculation yields the next proposition; for details see [MNO, Section 3.11]. In this proposition the elements $R(u, v)$ and $\tilde{R}(u, v)$ of $\operatorname{End}\left(\mathbb{C}^{N}\right)^{\otimes 2}(u, v)$ are identified with the elements $R(u, v) \otimes 1$ and $\tilde{R}(u, v) \otimes 1$ of $\operatorname{End}\left(\mathbb{C}^{N}\right)^{\otimes 2} \otimes \mathrm{U}(\mathfrak{g})(u, v)$ respectively. We will keep using this convention in the present section for simplicity.

Proposition 3.1. We have the relation in the algebra $\operatorname{End}\left(\mathbb{C}^{N}\right)^{\otimes 2} \otimes \mathrm{U}(\mathfrak{g})(u, v)$

$$
R(u, v) F_{1}(u) \tilde{R}(u, v) F_{2}(v)=F_{2}(v) \tilde{R}(u, v) F_{1}(u) R(u, v) .
$$

We will also use various embeddings of the algebra End $\left(\mathbb{C}^{N}\right)^{\otimes 2}$ into $\operatorname{End}\left(\mathbb{C}^{N}\right)^{\otimes m}$ with $m \geqslant 2$. For any $1 \leqslant p<q \leqslant m$ and $Y \in \operatorname{End}\left(\mathbb{C}^{N}\right)^{\otimes 2}$ we will denote

$$
Y_{p q}=\left(\iota_{p} \otimes \iota_{q}\right)(Y) \in \operatorname{End}\left(\mathbb{C}^{N}\right)^{\otimes m}
$$

In this section the number $m$ of the tensor factors will also vary, but $\mu$ will be always one of the two partitions $\left(1^{m}\right),(m)$. Let $A_{m}$ and $B_{m}$ be the elements of End $\left(\mathbb{C}^{N}\right)^{\otimes m}$ corresponding to antisymmetrization and symmetrization in the tensor product $\left(\mathbb{C}^{N}\right)^{\otimes m}$. They are normalized so that $A_{m}^{2}=A_{m}$ and $B_{m}^{2}=B_{m}$. Introduce the rational function $F_{\mu}(u)$ valued in $\operatorname{End}\left(\mathbb{C}^{N}\right)^{\otimes m} \otimes \mathrm{U}(\mathfrak{g})$ as follows:

$$
\begin{gathered}
F_{\left(1^{m}\right)}(u)=\left(A_{m} \otimes 1\right) \cdot \prod_{q=1}^{m}\left(\left(1+\frac{Q_{1 q}+\ldots+Q_{q-1, q}}{2 u-q+1}\right) \otimes 1\right) F_{q}(u-q+1), \\
F_{(m)}(u)=\left(B_{m} \otimes 1\right) \cdot \prod_{q=1}^{m}\left(\left(1+\frac{Q_{1 q}+\ldots+Q_{q-1, q}}{2 u+q-1}\right) \otimes 1\right) F_{q}(u+q-1) ;
\end{gathered}
$$

we will always arrange the (non-commuting) factors corresponding to the indices $q=1, \ldots, m$ from left to right.

Let us now choose the classical point $u_{\mu} \in \mathbb{C}$ as

$$
u_{\left(1^{m}\right)}=\frac{m}{2}-\eta, \quad u_{(m)}=-\frac{m}{2}-\eta
$$


Observe that the function $F_{\mu}(u)$ has no pole at $u=u_{\mu}$ when $\mathfrak{g}=\mathfrak{s o}_{N}, \mu=(m)$ or $\mathfrak{g}=\mathfrak{s p}_{N}, \mu=\left(1^{m}\right)$. Further, when $\mathfrak{g}=\mathfrak{s o}_{N}, \mu=\left(1^{m}\right)$ or $\mathfrak{g}=\mathfrak{s p}_{N}, \mu=(m)$ the order of the pole of $F_{\mu}(u)$ at $u=u_{\mu}$ does not exceed 1. So the following definition of the normalizing factor $\varphi_{\mu}(u) \in \mathbb{C}(u)$ makes the product $\varphi_{\mu}(u) \cdot F_{\mu}(u)$ regular at $u=u_{\mu}$ :

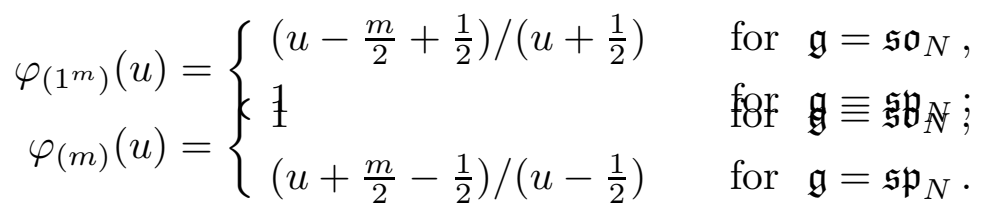

Let $\operatorname{tr}$ be the standard trace on $\operatorname{End}\left(\mathbb{C}^{N}\right)^{\otimes m}$. The following twin theorems constitute the main result of this section; cf. [O1, Theorem 1.3] and [N2, Theorem 5.3].

Theorem 3.2. For $\mu=\left(1^{2 k}\right)$ the value of $\varphi_{\mu}(u) \cdot(\operatorname{tr} \otimes \mathrm{id})\left(F_{\mu}(u)\right)$ at $u=u_{\mu}$ is $C_{k}$.

Theorem 3.3. For $\mu=(2 k)$ the value of $\varphi_{\mu}(u) \cdot(\operatorname{tr} \otimes \mathrm{id})\left(F_{\mu}(u)\right)$ at $u=u_{\mu}$ is $D_{k}$.

We will now present the proofs of both theorems as a chain of propositions.

Proposition 3.4. For each $\mu=\left(1^{m}\right),(m)$ we have $(\operatorname{tr} \otimes \mathrm{id})\left(F_{\mu}(u)\right) \in \mathrm{Z}(\mathfrak{g})(u)$.

Proof. Regard the group $G$ as a subgroup in $G L_{N} \subset \operatorname{End}\left(\mathbb{C}^{N}\right)$. Consider the adjoint action ad of the group $G$ in the enveloping algebra $\mathrm{U}(\mathfrak{g})$. Observe that by the definition (3.1) for any element $g \in G$ we have

$$
(\mathrm{id} \otimes \operatorname{ad} g)(F)=(g \otimes 1) \cdot F \cdot\left(g^{-1} \otimes 1\right) .
$$

Each of the elements $A_{m}, B_{m}$ and $Q_{p q}$ in $\operatorname{End}\left(\mathbb{C}^{N}\right)^{\otimes m}$ commutes with $g^{\otimes m}$. Thus

$$
(\mathrm{id} \otimes \operatorname{ad} g)\left(F_{\mu}(u)\right)=\left(g^{\otimes m} \otimes 1\right) \cdot F_{\mu}(u) \cdot\left(\left(g^{-1}\right)^{\otimes m} \otimes 1\right) .
$$

Hence

$$
(\operatorname{tr} \otimes \operatorname{ad} g)\left(F_{\mu}(u)\right)=(\operatorname{tr} \otimes \mathrm{id})\left(F_{\mu}(u)\right)
$$

The proof of the next proposition is based on the following simple lemma. Consider the product in $\operatorname{End}\left(\mathbb{C}^{N}\right)^{\otimes 3}(u, v, w)$

$$
R_{12}(u, v) \tilde{R}_{13}(u, w) \tilde{R}_{23}(v, w)=\tilde{R}_{23}(v, w) \tilde{R}_{13}(u, w) R_{12}(u, v) .
$$

The equality in (3.10) can be verified directly. Notice that the factor $\tilde{R}_{23}(v, w)$ at either side of this equality has a pole at $v+w=0$. Yet here is the simple lemma.

Lemma 3.5. The restrictions of (3.10) to the two sets of $(u, v, w)$ where $v=u \pm 1$, are regular at $v+w=0$.

Proof. Due to the relation $Q_{13} Q_{23}=P_{12} Q_{23}$ we have the equality

$$
R_{12}(u, u \pm 1) \tilde{R}_{13}(u, w) \tilde{R}_{23}(u \pm 1, w)=\left(1 \pm P_{12}\right) \cdot\left(1+\frac{Q_{13}+Q_{23}}{u+w}\right) .
$$

The latter rational function of $u, w$ is manifestly regular at $w=-u \mp 1$

Let us identify the elements $A_{m-1}$ and $B_{m-1}$ of the algebra $\operatorname{End}\left(\mathbb{C}^{N}\right)^{\otimes(m-1)}$ with their images under the natural embedding

$$
\operatorname{End}\left(\mathbb{C}^{N}\right)^{\otimes(m-1)} \rightarrow \operatorname{End}\left(\mathbb{C}^{N}\right)^{\otimes m}: X \mapsto X \otimes 1
$$

In the next proposition we as always arrange non-commuting factors corresponding to the indices $p=1, \ldots, m-1$ from left to right. 
Proposition 3.6. We have the equalities in $\operatorname{End}\left(\mathbb{C}^{N}\right)^{\otimes m}(u)$

$$
\begin{aligned}
& A_{m-1} \cdot \prod_{p=1}^{m-1} \tilde{R}_{p m}(u-p+1, u-m+1)=A_{m-1} \cdot\left(1+\frac{Q_{1 m}+\ldots+Q_{m-1, m}}{2 u-m+1}\right), \\
& B_{m-1} \cdot \prod_{p=1}^{m-1} \tilde{R}_{p m}(u+p-1, u+m-1)=B_{m-1} \cdot\left(1+\frac{Q_{1 m}+\ldots+Q_{m-1, m}}{2 u+m-1}\right) .
\end{aligned}
$$

Proof. We will give the proof of the first equality, the proof of the second is quite similar. Denote by $A(2 u)$ the rational function of $2 u$ at the left hand side of the first relation. The value of this function at $2 u=\infty$ is $A_{m-1}$. Moreover, its residue at $2 u=m-1$ is $Q_{1 m}+\ldots+Q_{m-1, m}$. So to prove the first relation it suffices to show that $A(2 u)$ has no poles except for a simple pole at $2 u=m-1$. Let an index $p \in\{2, \ldots, m-1\}$ be fixed. The corresponding factor $\tilde{R}_{p m}(u-p+1, u-m+1)$ in $A(2 u)$ has a pole at $2 u=p+m-2$. But when estimating from above the order of the pole at $2 u=p+m-2$ of $A(2 u)$, that factor does not count. Indeed, the element $A_{m-1} \in \operatorname{End}\left(\mathbb{C}^{N}\right)^{\otimes m}$ is divisible on the right by $R_{p-1, p}(u-p+2, u-p+1)$. Any factor in $A(2 u)$ corresponding to an index less than $p-1$ commutes with $R_{p-1, p}(u-p+2, u-p+1)$. But the product

$$
R_{p-1, p}(u-p+2, u-p+1) \tilde{R}_{p-1, m}(u-p+2, u-m+1) \tilde{R}_{p m}(u-p+1, u-m+1)
$$

has no pole at $2 u=p+m-2$ due to Lemma 3.5

We will make use of the well known decompositions of the elements $A_{m}$ and $B_{m}$

$$
\begin{aligned}
& A_{m}=\frac{1}{m !} \cdot \prod_{p=1}^{m-1} R_{p m}(1-p, 1-m) \ldots R_{p, p+1}(1-p,-p), \\
& B_{m}=\frac{1}{m !} \cdot \prod_{p=1}^{m-1} R_{p m}(p-1, m-1) \ldots R_{p, p+1}(p-1,+p)
\end{aligned}
$$

in $\operatorname{End}\left(\mathbb{C}^{N}\right)^{\otimes m}$. They can be easily verified by the induction on $m$; see for instance [MNO, Section 2.3]. Next proposition provides an alternative definition for each of the functions $F_{\left(1^{m}\right)}(u)$ and $F_{(m)}(u)$. This definition was motivated by [C1,S2].

Proposition 3.7. We have the equalities in $\operatorname{End}\left(\mathbb{C}^{N}\right)^{\otimes m} \otimes \mathrm{U}(\mathfrak{g})(u)$

$$
\begin{gathered}
F_{\left(1^{m}\right)}(u)=\left(A_{m} \otimes 1\right) \cdot \prod_{q=1}^{m}\left(\prod_{p=1}^{q-1} \tilde{R}_{p q}(u-p+1, u-q+1)\right) F_{q}(u-q+1), \\
F_{(m)}(u)=\left(B_{m} \otimes 1\right) \cdot \prod_{q=1}^{m}\left(\prod_{p=1}^{q-1} \tilde{R}_{p q}(u+p-1, u+q-1)\right) F_{q}(u+q-1) .
\end{gathered}
$$

Proof. It suffices to show that at the right hand sides of the above formulas for the rational functions $F_{\left(1^{m}\right)}(u)$ and $F_{(m)}(u)$ one can replace the ordered products

$$
\prod_{p=1}^{m-1} \tilde{R}_{p m}(u-p+1, u-m+1) \quad \text { and } \prod_{p=1}^{m-1} \tilde{R}_{p m}(u+p-1, u+m-1)
$$


by

$$
1+\frac{Q_{1 m}+\ldots+Q_{m-1, m}}{2 u-m+1} \quad \text { and } \quad 1+\frac{Q_{1 m}+\ldots+Q_{m-1, m}}{2 u+m-1}
$$

respectively. Using the decompositions (3.11),(3.12) for $m-1$ instead of $m$ and the relations $(3.6),(3.10)$ one can insert respectively $A_{m-1}$ and $B_{m-1}$ right after the factors $F_{m-1}(u-m+2)$ and $F_{m-1}(u+m-2)$ in the above formulas for $F_{\left(1^{m}\right)}(u)$ and $F_{(m)}(u)$. Here we also use the equalities $A_{m-1}^{2}=A_{m-1}$ and $B_{m-1}^{2}=B_{m-1}$. Now the required statement follows directly from Proposition 3.6

The main part of our proof of Theorems 3.2 and 3.3 will employ Corollary 2.5. As in Section 2 , let $\lambda$ be any partition with $\ell(\lambda) \leqslant n$. Let $V_{\lambda}$ be the corresponding irreducible $\mathfrak{g}$-module. Put $l=|\lambda|$. We will use the classical realization of $V_{\lambda}$ as a submodule in $\left(\mathbb{C}^{N}\right)^{\otimes l}$ consisting of traceless tensors. Recall that a vector $\xi \in\left(\mathbb{C}^{N}\right)^{\otimes l}$ is called traceless if $Q_{r s} \cdot \xi=0$ whenever $1 \leqslant r<s \leqslant l$. The subspace in $\left(\mathbb{C}^{N}\right)^{\otimes l}$ formed by all such vectors will be denoted by $V$.

Let $U_{\lambda}$ be the irreducible module over the Lie algebra $\mathfrak{g l}_{N}$ corresponding to the partition $\lambda$. Fix any embedding of the $\mathfrak{g l}_{N}$-module $U_{\lambda}$ to $\left(\mathbb{C}^{N}\right)^{\otimes l}$. The subspace $U_{\lambda} \cap V$ in $U_{\lambda}$ is preserved by the action of the subalgebra $\mathfrak{g} \subset \mathfrak{g l}_{N}$. For $\mathfrak{g}=\mathfrak{s o}_{2 n+1}, \mathfrak{s p}_{2 n}$ this subspace is isomorphic to $V_{\lambda}$ as $\mathfrak{g}$-module. For $\mathfrak{g}=\mathfrak{s o}_{2 n}$ it is isomorphic to $V_{\lambda}$ only if $\lambda_{n}=0$. Otherwise $U_{\lambda} \cap V$ splits into direct sum of the $\mathfrak{s o}_{2 n}$-modules $V_{\lambda}$ and $V_{\lambda^{*}}$. All these statements are contained in [W, Section V.C].

Our proof of Theorems 3.2 and 3.3 will be based on another simple lemma. Put

$$
E(u)=-u+\sum_{i j} E_{i j} \otimes E_{j i} \in \operatorname{End}\left(\mathbb{C}^{N}\right) \otimes \mathrm{U}\left(\mathfrak{g l}_{N}\right)[u] .
$$

Let

$$
\tilde{E}(u)=-u+\sum_{i j} \varepsilon_{i j} \cdot E_{i j} \otimes E_{-i,-j} \in \operatorname{End}\left(\mathbb{C}^{N}\right) \otimes \mathrm{U}\left(\mathfrak{g l}_{N}\right)[u]
$$

be the element obtained from $E(u)$ by applying the transposition with respect to the bilinear form $\langle$,$\rangle in the tensor factor \operatorname{End}\left(\mathbb{C}^{N}\right)$. Then consider the element

$$
\frac{\tilde{E}(\eta-u) E(\eta+u)}{u-\eta} \in \operatorname{End}\left(\mathbb{C}^{N}\right) \otimes \mathrm{U}\left(\mathfrak{g l}_{N}\right)(u) .
$$

We have the standard representation $\mathrm{U}\left(\mathfrak{g l}_{N}\right) \rightarrow \operatorname{End}\left(\mathbb{C}^{N}\right)^{\otimes l}$ so the element $(3.13)$ acts in the space $\mathbb{C}^{N} \otimes\left(\mathbb{C}^{N}\right)^{\otimes l}$. The element $F(u) \in \operatorname{End}\left(\mathbb{C}^{N}\right) \otimes \mathrm{U}(\mathfrak{g})[u]$ also acts in the space $\mathbb{C}^{N} \otimes\left(\mathbb{C}^{N}\right)^{\otimes l}$ and the latter action preserves the subspace $\mathbb{C}^{N} \otimes V$. Here is the lemma; cf. [O] and [MNO, Section 3.5].

Lemma 3.8. Action of the element $F(u)$ in $\mathbb{C}^{N} \otimes V$ coincides with that of (3.13).

Proof. Consider the image of $F(u) \in \operatorname{End}\left(\mathbb{C}^{N}\right) \otimes \mathrm{U}(\mathfrak{g})[u]$ in $\operatorname{End}\left(\mathbb{C}^{N}\right)^{\otimes(l+1)}[u]$ under the representation $\mathrm{U}(\mathfrak{g}) \rightarrow \operatorname{End}\left(\mathbb{C}^{N}\right)^{\otimes l}$. By (3.1) and (3.3) this image equals

$$
P_{12}+\ldots+P_{1, l+1}-Q_{12}-\ldots-Q_{1, l+1}-u-\eta .
$$

On the other hand, the image of the element $(3.13)$ in $\operatorname{End}\left(\mathbb{C}^{N}\right)^{\otimes(l+1)}(u)$ under the representation $\mathrm{U}\left(\mathfrak{g l}_{N}\right) \rightarrow \operatorname{End}\left(\mathbb{C}^{N}\right)^{\otimes l}$ is

$$
\left(1+\frac{Q_{12}+\ldots+Q_{1, l+1}}{u-\eta}\right) \cdot\left(P_{12}+\ldots+P_{1, l+1}-u-\eta\right)
$$


Using the relation (3.4) and definition of $\eta$ one shows that the product (3.15) equals (3.14) plus the sum

$$
\sum_{r \neq s} \frac{Q_{1, r+1} P_{1, s+1}}{u-\eta} .
$$

Since $Q_{1, r+1} P_{1, s+1}=P_{1, s+1} Q_{s+1, r+1}$ for $r \neq s$, the restriction of the latter sum to $\mathbb{C}^{N} \otimes V$ vanishes identically

Similarly to $(3.5)$, for $p=1, \ldots, m$ denote respectively by $E_{p}(u), \tilde{E}_{p}(u)$ the images in $\operatorname{End}\left(\mathbb{C}^{N}\right)^{\otimes m} \otimes \mathrm{U}\left(\mathfrak{g l}_{N}\right)[u]$ of $E(u), \tilde{E}(u)$ under the embedding $\iota_{p} \otimes \mathrm{id}$.

Proposition 3.9. We have the relations in the algebra End $\left(\mathbb{C}^{N}\right)^{\otimes 2} \otimes \mathbf{U}\left(\mathfrak{g l}_{N}\right)(u, v)$

$$
\begin{aligned}
R(u, v) E_{1}(u) E_{2}(v) & =E_{2}(v) E_{1}(u) R(u, v), \\
R(u, v) \tilde{E}_{1}(-u) \tilde{E}_{2}(-v) & =\tilde{E}_{2}(-v) \tilde{E}_{1}(-u) R(u, v), \\
\tilde{E}_{1}(-u) \tilde{R}(u, v) E_{2}(v) & =E_{2}(v) \tilde{R}(u, v) \tilde{E}_{1}(-u) .
\end{aligned}
$$

Proof. As well as (3.6), the relation (3.16) can be verified directly. Relation (3.17) is obtained from (3.16) by applying in the tensor factor $\mathrm{U}\left(\mathfrak{g l}_{N}\right)$ the automorphism $E_{i j} \mapsto-\varepsilon_{i j} \cdot E_{-j,-i}$. By applying to (3.16) the transposition with respect to $\langle$, in the fisrt tensor factor of $\operatorname{End}\left(\mathbb{C}^{N}\right)^{\otimes 2}$ we get (3.18)

We will need two more propositions. The first of them implies the classical Capelli identity $[\mathrm{C}]$. The second proposition implies the identity from [N1, Example 2] mentioned in Section 1. Observe that the $m$-th Capelli element of $\mathrm{Z}\left(\mathfrak{g l}_{N}\right)$ is the image under the map $\operatorname{tr} \otimes$ id of the element from $\operatorname{End}\left(\mathbb{C}^{N}\right)^{\otimes m} \otimes \mathrm{U}\left(\mathfrak{g l}_{N}\right)$

$$
\left(A_{m} \otimes 1\right) \cdot E_{1}(0) E_{2}(-1) \ldots E_{m}(1-m)
$$

see (1.3). Similarly, the element (1.5) with $k=m$ is the image under $\operatorname{tr} \otimes$ id of

$$
\left(B_{m} \otimes 1\right) \cdot E_{1}(0) E_{2}(1) \ldots E_{m}(m-1) .
$$

In proofs of both propositions we follow [M2]. For further details see [N2, Section 5].

Proposition 3.10. The element (3.19) of $\operatorname{End}\left(\mathbb{C}^{N}\right)^{\otimes m} \otimes \mathrm{U}\left(\mathfrak{g l}_{N}\right)$ vanishes in every representation $\mathrm{U}\left(\mathfrak{g l}_{N}\right) \rightarrow \operatorname{End}\left(U_{\lambda}\right)$ where $\ell(\lambda)<m$.

Proposition 3.11. The element (3.20) of $\operatorname{End}\left(\mathbb{C}^{N}\right)^{\otimes m} \otimes \mathrm{U}\left(\mathfrak{g l}_{N}\right)$ vanishes in every representation $\mathrm{U}\left(\mathfrak{g l}_{N}\right) \rightarrow \operatorname{End}\left(U_{\lambda}\right)$ where $\lambda_{1}<m$.

Proofs. We give only the proof of Proposition 3.10. The proof of Proposition 3.11 is similar and will be omitted. We will show by induction on $m$ that the image in End $\left(\mathbb{C}^{N}\right)^{(m+l)}$ of $(3.19)$ under the representation $\mathrm{U}\left(\mathfrak{g l}_{N}\right) \rightarrow \operatorname{End}\left(\mathbb{C}^{N}\right)^{\otimes l}$ equals

$$
A_{m} \cdot \sum_{r_{1} \ldots r_{m}} P_{1, m+r_{1}} \ldots P_{m, m+r_{m}}
$$

where all the indices $r_{1}, \ldots, r_{m} \in\{1, \ldots, l\}$ are pairwise distinct. The sum in (3.21) vanishes when $l<m$. When $l \geqslant m$ but $\ell(\lambda)<m$ the required vanishing property will follow from the Young decomposition of the $\mathfrak{g l}_{N}$-module $U_{\left(1^{m}\right)} \otimes\left(\mathbb{C}^{N}\right)^{\otimes(l-m)}$ into irreducible components. 
By definition the image in $\operatorname{End}\left(\mathbb{C}^{N}\right)^{(m+l)}[u]$ of $E_{p}(u) \in \operatorname{End}\left(\mathbb{C}^{N}\right)^{\otimes m} \otimes \mathrm{U}\left(\mathfrak{g l}_{N}\right)$ under the representation $\mathrm{U}\left(\mathfrak{g l}_{N}\right) \rightarrow \operatorname{End}\left(\mathbb{C}^{N}\right)^{\otimes l}$ is

$$
-u+\sum_{r} P_{p, m+r}
$$

where the sum is taken over $r=1, \ldots, l$. In particular, this provides the base $m=1$ for our induction. Now suppose that $m>1$. By the inductive assumption the image in $\operatorname{End}\left(\mathbb{C}^{N}\right)^{\otimes(m+l)}$ of the element (3.19) equals

$$
\begin{gathered}
A_{m} \cdot \sum_{r_{1} \ldots r_{m-1}} P_{1, m+r_{1}} \ldots P_{m-1, m+r_{m-1}} \cdot \sum_{r} P_{m, m+r}+ \\
(m-1) \cdot A_{m} \cdot \sum_{r_{1} \ldots r_{m-1}} P_{1, m+r_{1}} \ldots P_{m-1, m+r_{m-1}}
\end{gathered}
$$

where all the indices $r_{1}, \ldots, r_{m-1} \in\{1, \ldots, l\}$ are pairwise distinct. Here the expression in the second line can be replaced by

$$
-A_{m} \cdot \sum_{p=1}^{m-1} P_{p m} \cdot \sum_{r_{1} \ldots r_{m-1}} P_{1, m+r_{1}} \ldots P_{m-1, m+r_{m-1}}
$$

But the latter expression can be rewritten as

$$
-A_{m} \cdot \sum_{r_{1} \ldots r_{m-1}} P_{1, m+r_{1}} \ldots P_{m-1, m+r_{m-1}} \cdot \sum_{p=1}^{m-1} P_{m, m+r_{p}} .
$$

Hence after this replacement the sum (3.22) becomes evidently equal to (3.21)

Note that Proposition 3.10 can be reformulated in the following way.

Corollary 3.12. The element of $\operatorname{End}\left(\mathbb{C}^{N}\right)^{\otimes m} \otimes \mathrm{U}\left(\mathfrak{g l}_{N}\right)$

$$
\left(A_{m} \otimes 1\right) \cdot \tilde{E}_{1}(1-m) \ldots \tilde{E}_{m-1}(-1) \tilde{E}_{m}(0)
$$

vanishes in every representation $\mathrm{U}\left(\mathfrak{g l}_{N}\right) \rightarrow \operatorname{End}\left(U_{\lambda}\right)$ where $\ell(\lambda)<m$.

Proof. By using the decomposition (3.11) and the relation (3.16) the element (3.19) of the algebra End $\left(\mathbb{C}^{N}\right)^{\otimes m} \otimes \mathrm{U}\left(\mathfrak{g l}_{N}\right)$ can be rewritten as

$$
E_{m}(1-m) \ldots E_{2}(-1) E_{1}(0) \cdot\left(A_{m} \otimes 1\right) \text {. }
$$

Applying to (3.24) the transposition in each tensor factor of $\operatorname{End}\left(\mathbb{C}^{N}\right)^{\otimes m}$ relative to $\langle$,$\rangle we get$

$$
\left(A_{m} \otimes 1\right) \cdot \tilde{E}_{m}(1-m) \ldots \tilde{E}_{2}(-1) \tilde{E}_{1}(0) .
$$

Conjugate the latter element by $P_{m} \otimes 1$, where $P_{m} \in \operatorname{End}\left(\mathbb{C}^{N}\right)^{\otimes m}$ corresponds to the operator

$$
e_{i_{1}} \otimes e_{i_{2}} \otimes \ldots \otimes e_{i_{m}} \mapsto e_{i_{m}} \otimes \ldots \otimes e_{i_{2}} \otimes e_{i_{1}}
$$

in $\left(\mathbb{C}^{N}\right)^{\otimes m}$. Then we obtain exactly the element $(3.24)$

By using the decomposition (3.12) and again Proposition 3.9, we obtain a corollary to Proposition 3.11 parallel to Corollary 3.12. 
Corollary 3.13. The element of $\operatorname{End}\left(\mathbb{C}^{N}\right)^{\otimes m} \otimes \mathrm{U}\left(\mathfrak{g l}_{N}\right)$

$$
\left(B_{m} \otimes 1\right) \cdot \tilde{E}_{1}(m-1) \ldots \tilde{E}_{m-1}(1) \tilde{E}_{m}(0)
$$

vanishes in every representation $\mathrm{U}\left(\mathfrak{g l}_{N}\right) \rightarrow \operatorname{End}\left(U_{\lambda}\right)$ where $\lambda_{1}<m$.

If we replace each appearance of the function $F(u)$ in the definitions of $F_{\left(1^{m}\right)}(u)$ and $F_{(m)}(u)$ by $(3.13)$, we obtain the functions valued in $\operatorname{End}\left(\mathbb{C}^{N}\right)^{\otimes m} \otimes \mathrm{U}\left(\mathfrak{g l}_{N}\right)$

$$
\begin{aligned}
& \left(A_{m} \otimes 1\right) \cdot \prod_{q=1}^{m}\left(\left(1+\frac{Q_{1 q}+\ldots+Q_{q-1, q}}{2 u-q+1}\right) \otimes 1\right) \frac{\tilde{E}_{q}(\eta-u+q-1) E_{q}(\eta+u-q+1)}{u-q+1-\eta}, \\
& \left(B_{m} \otimes 1\right) \cdot \prod_{q=1}^{m}\left(\left(1+\frac{Q_{1 q}+\ldots+Q_{q-1, q}}{2 u+q-1}\right) \otimes 1\right) \frac{\tilde{E}_{q}(\eta-u-q+1) E_{q}(\eta+u+q-1)}{u+q-1-\eta} .
\end{aligned}
$$

We will denote these rational functions by $G_{\left(1^{m}\right)}(u)$ and $G_{(m)}(u)$ respectively.

Now for either of the two partitions $\mu=\left(1^{2 k}\right),(2 k)$ of $m=2 k$ take the value of

$$
\varphi_{\mu}(u) \cdot F_{\mu}(u) \in \operatorname{End}\left(\mathbb{C}^{N}\right)^{\otimes m} \otimes \mathrm{U}(\mathfrak{g})(u)
$$

at $u=u_{\mu}$. Let us consider the image of this value with respect to the representation $\mathrm{U}(\mathfrak{g}) \rightarrow \operatorname{End}\left(V_{\lambda}\right)$. For $\mu=\left(1^{2 k}\right)$ we will show that this image is zero when $\ell(\lambda)<k$. For $\mu=(2 k)$ we will show that this image is zero when $\lambda_{1}<k$. Thanks to Lemma 3.8 it suffices to prove the next twin propositions.

Proposition 3.14. For $\mu=\left(1^{2 k}\right)$ the value of the function $\varphi_{\mu}(u) \cdot G_{\mu}(u)$ at $u=u_{\mu}$ vanishes in every representation $\mathrm{U}\left(\mathfrak{g l}_{N}\right) \rightarrow \operatorname{End}\left(U_{\lambda}\right)$ with $\ell(\lambda)<k$.

Proposition 3.15. For $\mu=(2 k)$ the value of the function $\varphi_{\mu}(u) \cdot G_{\mu}(u)$ at $u=u_{\mu}$ vanishes in every representation $\mathrm{U}\left(\mathfrak{g l}_{N}\right) \rightarrow \operatorname{End}\left(U_{\lambda}\right)$ with $\lambda_{1}<k$.

Proofs. We will give the proof of Proposition 3.14; the proof of Proposition 3.15 is similar and will be omitted. We will prove that the function $G_{\left(1^{2 k}\right)}(u)$ equals

$$
\begin{gathered}
\left(A_{2 k} \otimes 1\right) \cdot \prod_{q=1}^{2 k} \tilde{E}_{q}(\eta-u+q-1) \times \\
\prod_{q=1}^{2 k}\left(\left(1+\frac{Q_{1 q}+\ldots+Q_{q-1, q}}{2 u-q+1}\right) \otimes 1\right) \cdot \prod_{q=1}^{2 k} \frac{1}{u-q+1-\eta} \times \\
\left(A_{2 k} \otimes 1\right) \cdot \prod_{q=1}^{2 k} E_{q}(\eta+u-q+1)
\end{gathered}
$$

where the non-commuting factors corresponding to the indices $q=1, \ldots, 2 k$ are as usual arranged from left to right. Note that we have defined $u_{\left(1^{2 k}\right)}=k-\eta$. But in the third line of (3.25) we get the function valued in $\operatorname{End}\left(\mathbb{C}^{N}\right)^{\otimes 2 k} \otimes \mathrm{U}\left(\mathfrak{g l}_{N}\right)$

$\left(A_{2 k} \otimes 1\right) \cdot E_{1}(u+\eta) \ldots E_{k}(u+\eta-k+1) \cdot E_{k+1}(u+\eta-k) \ldots E_{2 k}(u+\eta-2 k+1)$. 
Its value at $u=k-\eta$ vanishes in every representation $\mathrm{U}\left(\mathfrak{g l}_{N}\right) \rightarrow \operatorname{End}\left(U_{\lambda}\right)$ with $\ell(\lambda)<k$. To see this consider the last $k$ factors in the above product and apply Proposition 3.10 to $m=k$.

Suppose that $\mathfrak{g}=\mathfrak{s o}_{N}$. Then $\eta=\frac{1}{2}$ and $u_{\left(1^{2 k}\right)}=k-\frac{1}{2}$. Observe that $Q_{p q} \cdot A_{2 k}=0$ for any $1 \leqslant p<q \leqslant 2 k$. Therefore the first product in the second line of $(3.25)$ can be replaced by 1 . The second product in that line has a pole at $u=k-\frac{1}{2}$ of degree 1 . Thus we will obtain Proposition 3.14 for $\mathfrak{g}=\mathfrak{s o}_{N}$ since in this case

$$
\varphi_{\left(1^{2 k}\right)}(u)=\left(u-k+\frac{1}{2}\right) /\left(u+\frac{1}{2}\right) .
$$

Now suppose that $\mathfrak{g}=\mathfrak{s p}_{N}$. Then $\eta=-\frac{1}{2}, u_{\left(1^{2 k}\right)}=k+\frac{1}{2}$ while $\varphi_{\left(1^{2 k}\right)}(u)=1$. If $k=1$ then the rational function in second line of (3.25) has no pole at $u=\frac{3}{2}$; so we get Proposition 3.14 by considering the product in the third line of $(3.25)$ only. Assume that $k \geqslant 2$. Then the function in the second line of (3.25) has a pole at $u=k+\frac{1}{2}$ of degree at most 1. But in the first line of (3.25) we then get

$$
\begin{gathered}
\left(A_{2 k} \otimes 1\right) \cdot \tilde{E}_{1}\left(-\frac{1}{2}-u\right) \tilde{E}_{2}\left(\frac{1}{2}-u\right) \cdot \tilde{E}_{3}\left(\frac{3}{2}-u\right) \ldots \tilde{E}_{k+2}\left(k+\frac{1}{2}-u\right) \times \\
\tilde{E}_{k+3}\left(k+\frac{3}{2}-u\right) \ldots \tilde{E}_{2 k}\left(2 k-\frac{3}{2}-u\right) .
\end{gathered}
$$

The value of (3.26) at $u=k+\frac{1}{2}$ vanishes in any representation $\mathrm{U}\left(\mathfrak{g l}_{N}\right) \rightarrow \operatorname{End}\left(U_{\lambda}\right)$ with $\ell(\lambda)<k$. To see this take the last $k$ factors in the first line of (3.26) and apply Corollary 3.12 to $m=k$. Thus we will obtain Proposition 3.14 for $\mathfrak{g}=\mathfrak{s p}_{N}$.

It remains to show that the function $G_{\left(1^{2 k}\right)}(u)$ indeed equals (3.25). By using (3.11) and (3.17) we obtain for any $m$ the relation in $\operatorname{End}\left(\mathbb{C}^{N}\right)^{\otimes m} \otimes \mathrm{U}\left(\mathfrak{g l}_{N}\right)(u)$

$$
\left(A_{m} \otimes 1\right) \cdot \tilde{E}_{1}(u) \ldots \tilde{E}_{m}(u+m-1)=\tilde{E}_{m}(u+m-1) \ldots \tilde{E}_{1}(u) \cdot\left(A_{m} \otimes 1\right) .
$$

In particular, the expression in the first line of (3.25) is divisible on the right by $A_{2 k} \otimes 1$. But due to Proposition 3.6 we have

$$
\begin{gathered}
A_{2 k} \cdot \prod_{q=1}^{2 k}\left(1+\frac{Q_{1 q}+\ldots+Q_{q-1, q}}{2 u-q+1}\right)=A_{2 k} \cdot \prod_{q=1}^{2 k} A_{q-1}\left(1+\frac{Q_{1 q}+\ldots+Q_{q-1, q}}{2 u-q+1}\right) \\
=A_{2 k} \cdot \prod_{q=1}^{2 k}\left(\prod_{p=1}^{q-1} \tilde{R}_{p q}(u-p+1, u-q+1)\right) .
\end{gathered}
$$

So (3.25) equals

$$
\begin{gathered}
\left(A_{2 k} \otimes 1\right) \cdot \prod_{q=1}^{2 k} \tilde{E}_{q}(\eta-u+q-1) \times \\
\prod_{q=1}^{2 k}\left(\prod_{p=1}^{q-1} \tilde{R}_{p q}(u-p+1, u-q+1)\right) \cdot \prod_{q=1}^{2 k} \frac{1}{u-q+1-\eta} \times \\
\left(A_{2 k} \otimes 1\right) \cdot \prod_{q=1}^{2 k} E_{q}(\eta+u-q+1) .
\end{gathered}
$$

By using only (3.18) we can transform this expression to

$$
\left(A_{2 k} \otimes 1\right) \cdot \prod_{q=1}^{2 k}\left(\prod_{p=1}^{q-1} \tilde{R}_{p q}(u-p+1, u-q+1)\right) \frac{\tilde{E}_{q}(\eta-u+q-1) E_{q}(\eta+u-q+1)}{u-q+1-\eta}
$$


The latter expression equals $G_{\left(1^{2 k}\right)}(u)$. To prove this observe that the element (3.13) satisfies a relation in $\operatorname{End}\left(\mathbb{C}^{N}\right)^{\otimes 2} \otimes \mathrm{U}\left(\mathfrak{g l}_{N}\right)(u, v)$ similar to (3.6)

$$
\begin{aligned}
& R(u, v) \cdot \tilde{E}_{1}(\eta-u) E_{1}(\eta+u) \cdot \tilde{R}(u, v) \cdot \tilde{E}_{2}(\eta-v) E_{2}(\eta+v)= \\
& \tilde{E}_{2}(\eta-v) E_{2}(\eta+v) \cdot \tilde{R}(u, v) \cdot \tilde{E}_{1}(\eta-u) E_{1}(\eta+u) \cdot R(u, v)
\end{aligned}
$$

This relation can be easily deduced from $(3.16),(3.17),(3.18)$. So we can repeat the arguments from Proposition 3.7 for the function (3.13) instead of $F(u)$

Now for $\mu=\left(1^{2 k}\right)$ take the value of the function $\varphi_{\mu}(u) \cdot(\operatorname{tr} \otimes \mathrm{id})\left(F_{\mu}(u)\right)$ at $u=u_{\mu}$. Denote this value by $Z$. By Proposition 3.1 we have $Z \in \mathrm{Z}(\mathfrak{g})$. The degree of $Z$ with respect to the standard filtration on $\mathrm{U}(\mathfrak{g})$ does not exceed $2 k$ by the definition of $F_{\mu}(u)$. By Corollary 2.5 we obtain from Proposition 3.14 that $Z$ equals $C_{k}$ up to a scalar multiple. To complete the proof of Theorem 3.2 it remains to show that this multiple is actually 1 . Similarly, for $\mu=(2 k)$ due to Proposition 3.15 the value of $\varphi_{\mu}(u) \cdot(\operatorname{tr} \otimes \mathrm{id})\left(F_{\mu}(u)\right)$ at $u=u_{\mu}$ is $D_{k}$ up to a scalar multiple. So to complete the proof of Theorem 3.3 it remains to show that this multiple is also 1 .

For $\mu=\left(1^{2 k}\right),(2 k)$ take the image under the Harish-Chandra isomorphism

$$
\omega\left(\varphi_{\mu}(u) \cdot(\operatorname{tr} \otimes \mathrm{id})\left(F_{\mu}(u)\right)\right)
$$

Consider this image as a polynomial in $\lambda_{1}, \ldots, \lambda_{n}$ with the coefficients from $\mathbb{C}(u)$. The next two propositions complete the proofs of Theorems 3.2 and 3.3 respectively. They also justify our choice $(3.8),(3.9)$ of the normalizing factor $\varphi_{\mu}(u) \in \mathbb{C}(u)$.

Proposition 3.16. For $\mu=\left(1^{2 k}\right)$ the leading component of the polynomial (3.27) is $(-1)^{k} e_{k}\left(\lambda_{1}^{2}, \ldots, \lambda_{n}^{2}\right)$.

Proposition 3.17. For $\mu=(2 k)$ the leading component of the polynomial (3.27) is $h_{k}\left(\lambda_{1}^{2}, \ldots, \lambda_{n}^{2}\right)$.

Proofs. We shall give the proof of Proposition 3.16; the proof of Proposition 3.17 is similar and will be omitted. We will write $m=2 k$ for short. First let us prove that leading component of the polynomial (3.27) with $\mu=\left(1^{m}\right)$ does not depend on the parameter $u$. We will use the equality in $\operatorname{End}\left(\mathbb{C}^{N}\right)^{\otimes m} \otimes \mathrm{U}(\mathfrak{g})(u)$

$$
F_{\left(1^{m}\right)}(u)=\left(A_{m} \otimes 1\right) \cdot \prod_{q=1}^{m} F_{q}(u-q+1)\left(\left(1+\frac{Q_{q, q+1}+\ldots+Q_{q m}}{2 u-2 q+1}\right) \otimes 1\right)
$$

where the factors corresponding to the indices $q=1, \ldots, m$ are as usual arranged from left to right. It can be proved by using Proposition 3.7, see also Proposition 3.6. Denote by $A_{m-1}^{\prime}$ the image of the element $A_{m-1}$ with respect to the embedding

$$
\operatorname{End}\left(\mathbb{C}^{N}\right)^{\otimes(m-1)} \rightarrow \operatorname{End}\left(\mathbb{C}^{N}\right)^{\otimes m}: X \mapsto 1 \otimes X
$$

Let us use the well known decomposition $A_{m}=T_{m} \cdot A_{m-1}^{\prime}$ in $\operatorname{End}\left(\mathbb{C}^{N}\right)^{\otimes m}$ where

$$
T_{m}=\left(1-P_{12}-\ldots-P_{1 m}\right) / m
$$


By this decomposition and by the above formula for $F_{\left(1^{m}\right)}(u)$ that function equals

$$
\left(T_{m} \otimes 1\right) F_{1}(u)\left(\left(1+\frac{Q_{12}+\ldots+Q_{1 m}}{2 u-1}\right) \otimes 1\right) F_{\left(1^{m-1}\right)}^{\prime}(u-1)
$$

where $F_{\left(1^{m-1}\right)}^{\prime}(u)$ stands for the image of $F_{\left(1^{m-1}\right)}(u) \in \operatorname{End}\left(\mathbb{C}^{N}\right)^{\otimes(m-1)} \otimes \mathrm{U}(\mathfrak{g})(u)$ with respect to the embedding (3.28). Take the standard filtration on the algebra $\mathrm{U}(\mathfrak{g})$. Extend it to the algebra $\mathrm{U}(\mathfrak{g})(u)$ by setting $\operatorname{deg} u=0$. Denote

$$
\psi(u)= \begin{cases}\left(u+\frac{1}{2}\right) /\left(u-\frac{1}{2}\right) & \text { for } \mathfrak{g}=\mathfrak{s o}_{N} \\ 1 & \text { for } \mathfrak{g}=\mathfrak{s p}_{N} .\end{cases}
$$

Then the image in $\mathrm{U}(\mathfrak{g})(u)$ of the element (3.29) under $\operatorname{tr} \otimes$ id is equal to that of

$$
\psi(u) \cdot\left(T_{m} \otimes 1\right) F_{1}(u) F_{\left(1^{m-1}\right)}^{\prime}(u-1)
$$

plus lower degree terms. Indeed, due to (3.1) and (3.3) for any $r=2, \ldots, m$ the element

$$
\left(P_{1 r} \otimes 1\right) \cdot F_{1} \cdot\left(Q_{1 r} \otimes 1\right) \in \operatorname{End}\left(\mathbb{C}^{N}\right)^{\otimes m} \otimes \mathrm{U}(\mathfrak{g})
$$

is equal to $-F_{1} \cdot\left(Q_{1 r} \otimes 1\right)$ if $\mathfrak{g}=\mathfrak{s o}_{N}$ and to $F_{1} \cdot\left(Q_{1 r} \otimes 1\right)$ if $\mathfrak{g}=\mathfrak{s p}{ }_{N}$. Therefore

$$
\left(T_{m} \otimes 1\right) F_{1} \cdot\left(\left(1+\frac{Q_{12}+\ldots+Q_{1 m}}{2 u-1}\right) \otimes 1\right)=\psi(u) \cdot\left(T_{m} \otimes 1\right) F_{1}
$$

plus a certain element of End $\left(\mathbb{C}^{N}\right)^{\otimes m} \otimes \mathrm{U}(\mathfrak{g})(u)$ which is antisymmetric under the transposition in the first tensor factor $\operatorname{End}\left(\mathbb{C}^{N}\right)$ with respect to the bilinear form $\langle$,$\rangle . So the image of the latter element under the map \operatorname{tr} \otimes$ id is zero.

Let us now use the definition of $F_{\left(1^{m}\right)}(u)$ directly. By the properties of trace, the image of $F_{\left(1^{m}\right)}(u)$ under $\operatorname{tr} \otimes$ id will remain unchanged when we move $A_{m} \otimes 1$ to the rightmost position and then apply the conjugation by $\left(P_{12} \ldots P_{1 m}\right) \otimes 1$. After that we will decompose $A_{m}=T_{m} \cdot A_{m-1}^{\prime}$ and move $A_{m-1}^{\prime}$ to the leftmost position. The transformed expression for the element $F_{\left(1^{m}\right)}(u-1)$ can be written as

$$
F_{\left(1^{m-1}\right)}^{\prime}(u-1)\left(\left(1+\frac{Q_{12}+\ldots+Q_{1 m}}{2 u-m-1}\right) \otimes 1\right) F_{1}(u-m)\left(T_{m} \otimes 1\right) .
$$

But the image of the latter element under the map $\operatorname{tr} \otimes \mathrm{id}$ is equal to the image of

$$
\psi\left(u-\frac{m}{2}\right) \cdot F_{\left(1^{m-1}\right)}^{\prime}(u-1) F_{1}(u-m)\left(T_{m} \otimes 1\right)
$$

plus lower degree terms in $\mathrm{U}(\mathfrak{g})(u)$. Note that by the definition (3.8)

$$
\varphi_{\left(1^{m}\right)}(u) \cdot \psi(u)=\varphi_{\left(1^{m}\right)}(u-1) \cdot \psi\left(u-\frac{m}{2}\right) .
$$

Therefore by comparing the expressions (3.30) and (3.31) we obtain that the degree $m$ component of the polynomial (3.27) with $\mu=\left(1^{m}\right)$ does not change if we replace $u$ by $u-1$. As this component is a rational function in $u$, it must be constant in $u$.

To complete the proof of Proposition 3.16 it now suffices to determine the leading component of (3.27) with $\mu=\left(1^{m}\right)$ at $u \rightarrow \infty$. This can be done as follows. In 
the definition of $F_{\left(1^{m}\right)}(u)$ replace each factor $F_{q}(u-q+1)$ by $F_{q}$ and note that $\left(Q_{1 q}+\ldots+Q_{q-1, q}\right) /(2 u-q+1) \rightarrow 0$ when $u \rightarrow \infty$. Since $\varphi_{\left(1^{m}\right)}(u) \rightarrow 1$ when $u \rightarrow \infty$, it now remains to calculate the leading component of the polynomial

$$
\omega\left((\operatorname{tr} \otimes \mathrm{id})\left(\left(A_{m} \otimes 1\right) \cdot F_{1} \ldots F_{m}\right)\right)
$$

with $m=2 k$. This calculation is immediate, and yields $(-1)^{k} e_{k}\left(\lambda_{1}^{2}, \ldots, \lambda_{n}^{2}\right)$

\section{Capelli identity for the Lie algebra $\mathfrak{s o}_{N}$}

In this section we will keep the positive integer $N$ fixed. Let us now fix one more positive integer $m$. We will consider a natural representation of the enveloping algebra $\mathrm{U}\left(\mathfrak{s o}_{N}\right)$ by differential operators with polynomial coefficients on the space $\mathbb{C}^{m} \otimes \mathbb{C}^{N}$. We will give an explicit formula for the differential operator corresponding to the element $C_{k} \in \mathrm{Z}\left(\mathfrak{s o}_{N}\right)$ with $k=1, \ldots, n$. We will show that this formula may be regarded as an analogue for $\mathfrak{s o}_{N}$ of the Capelli identity [C] for $\mathfrak{g l}_{N}$.

As well as in Sections 2 and 3 here the indices $i$ and $j$ will run through the set $\{-n, \ldots,-1,1, \ldots, n\}$ if $N=2 n$ and through the set $\{-n, \ldots,-1,0,1, \ldots, n\}$ if $N=2 n+1$. The indices $a$ and $b$ will run through $\{1, \ldots, m\}$. Let $x_{a i}$ be independent commuting variables and $\partial_{a i}$ be the corresponding differentiations. Denote by $\mathcal{P}$ the ring of polynomials in the variables $x_{a i}$. Take the action (1.2) of the Lie algebra $\mathfrak{g l}_{N}$ on $\mathcal{P}$. We will use the embedding of the group $O_{N}$ into $G L_{N}$ from Section 2. Then restriction of the above action of $\mathfrak{g l}_{N}$ in $\mathcal{P}$ to $\mathfrak{s o}_{N}$ is

$$
F_{i j} \mapsto \sum_{a}\left(x_{a i} \partial_{a j}-x_{a,-j} \partial_{a,-i}\right)
$$

Denote by $\mathcal{P D}$ the ring of differential operators in $x_{a i}$ with polynomial coefficients. Then (4.1) determines a representation $\gamma: \mathrm{U}\left(\mathfrak{s o}_{N}\right) \rightarrow \mathcal{P D}$. We will give a formula for the image in $\mathcal{P D}$ of the element $C_{k} \in \mathrm{Z}\left(\mathfrak{s o}_{N}\right)$ with respect to $\gamma$.

By definition we have $F_{i,-j}=-F_{j,-i}$. Let $I$ be any set of indices $i$ with $2 k$ elements. Suppose these elements are $i_{1}<\ldots<i_{2 k}$. Take an element of $\mathrm{U}\left(\mathfrak{s o}_{N}\right)$

$$
\Phi_{I}=\sum_{\sigma} \frac{\operatorname{sgn}(\sigma)}{2^{k} k !} \cdot F_{i_{\sigma(1)},-i_{\sigma(2)}} \ldots F_{i_{\sigma(2 k-1)},-i_{\sigma(2 k)}}
$$

where $\sigma$ runs through the symmetric group $S_{2 k}$. The element (4.2) is the Pfaffian of the antisymmetric matrix $\left[F_{i_{p},-i_{q}}\right]$ with the indices $p, q=1, \ldots, 2 k$. Note that the factors of the monomial in the sum (4.2) do not commute in general. Still we will write

$$
\Phi_{I}=\operatorname{Pf}\left[F_{i_{p},-i_{q}}\right] ; \quad p, q=1, \ldots, 2 k .
$$

Let us denote $I^{*}=\left\{-i_{2 k}, \ldots,-i_{1}\right\}$. Further, let $A$ be any set of indices $a$ with $k$ elements. Suppose these elements are $a_{1}, \ldots, a_{k}$. Then denote in $\mathcal{P} \mathcal{D}$

$$
\Omega_{A I}=\sum_{J} \operatorname{sgn}\left(J J^{\prime}\right) \cdot \operatorname{det}\left[x_{a_{p} j_{q}}\right] \operatorname{det}\left[\partial_{a_{p},-j_{q}^{\prime}}\right]
$$

where the sum is taken over all partitions of $I$ into two subsets $J=\left\{j_{1}, \ldots, j_{k}\right\}$ and $J^{\prime}=\left\{j_{1}^{\prime}, \ldots, j_{k}^{\prime}\right\}$. Here the determinants are taken with respect to the indices $p, q=1, \ldots, k$ and $\operatorname{sgn}\left(J J^{\prime}\right)$ is the sign of the permutation $\left(j_{1}, j_{1}^{\prime}, \ldots, j_{k}, j_{k}^{\prime}\right)$ of the sequence $\left(i_{1}, \ldots, i_{2 k}\right)$. Next theorem provides an explicit formula for $\gamma\left(C_{k}\right)$. 
Theorem 4.1. We have the equalities respectively in $\mathrm{U}\left(\mathfrak{s o}_{N}\right)$ and $\mathcal{P} \mathcal{D}$

$$
C_{k}=(-1)^{k} \cdot \sum_{I} \Phi_{I} \Phi_{I^{*}} \quad \text { and } \quad \gamma\left(\Phi_{I}\right)=\sum_{A} \Omega_{A I}
$$

Proof. We start with verifying the second equality in (4.4). By definition $\Phi_{I}$ equals

$$
\begin{gathered}
\sum_{\sigma} \frac{\operatorname{sgn}(\sigma)}{2^{k} k !} \cdot\left(E_{i_{\sigma(1)},-i_{\sigma(2)}}-E_{i_{\sigma(2)},-i_{\sigma(1)}}\right) \ldots\left(E_{i_{\sigma(2 k-1)},-i_{\sigma(2 k)}}-E_{i_{\sigma(2 k)},-i_{\sigma(2 k-1)}}\right) \\
=\sum_{\sigma} \frac{\operatorname{sgn}(\sigma)}{k !} \cdot E_{i_{\sigma(1)},-i_{\sigma(2)}} \ldots E_{i_{\sigma(2 k-1)},-i_{\sigma(2 k)}}
\end{gathered}
$$

in $\mathrm{U}\left(\mathfrak{s o}_{N}\right) \subset \mathrm{U}\left(\mathfrak{g l}_{N}\right)$. Therefore the image $\gamma\left(\Phi_{I}\right) \in \mathcal{P} \mathcal{D}$ equals the sum

$$
\sum_{\sigma} \sum_{a_{1}, \ldots, a_{k}=1}^{m} \frac{\operatorname{sgn}(\sigma)}{k !} \cdot x_{a_{1} i_{\sigma(1)}} \partial_{a_{1},-i_{\sigma(2)}} \ldots x_{a_{k} i_{\sigma(2 k-1)}} \partial_{a_{k},-i_{\sigma(2 k)}} .
$$

Move each operator $\partial_{a_{p},-i_{\sigma(2 p)}}$ in every monomial of (4.5) to the right, commuting it consecutively with the multiplication operators $x_{a_{q} i_{\sigma(2 q-1)}}$ for $q=p+1, \ldots, k$. Let us start with the operator $\partial_{a_{1},-i_{\sigma(2)}}$. Take the commutator

$$
\left[\partial_{a_{1},-i_{\sigma(2)}}, x_{a_{2} i_{\sigma(3)}}\right]=\delta_{a_{1} a_{2}} \delta_{-i_{\sigma(2)}, i_{\sigma(3)}}
$$

The sum over $\sigma$ in (4.5) involves two commutators of this form with opposite signs. Let $\bar{\sigma} \in S_{2 k}$ is such that $\bar{\sigma}(r)=\sigma(r)$ for $r \neq 2,3$ and $\bar{\sigma}(2)=\sigma(3), \bar{\sigma}(3)=\sigma(2)$. Then $\operatorname{sgn}(\bar{\sigma})=-\operatorname{sgn}(\sigma)$ whilst $\left[\partial_{a_{1},-i_{\bar{\sigma}(2)}}, x_{a_{2} i_{\bar{\sigma}(3)}}\right]=\left[\partial_{a_{1},-i_{\sigma(2)}}, x_{a_{2} i_{\sigma(3)}}\right]$. So by repeating this argument we get

$$
\gamma\left(\Phi_{I}\right)=\sum_{\sigma} \sum_{a_{1}, \ldots, a_{k}=1}^{m} \frac{\operatorname{sgn}(\sigma)}{k !} \cdot x_{a_{1} i_{\sigma(1)}} \ldots x_{a_{k} i_{\sigma(2 k-1)}} \partial_{a_{1},-i_{\sigma(2)}} \ldots \partial_{a_{k},-i_{\sigma(2 k)}}
$$

This equality can be obviously rewritten as

$$
\gamma\left(\Phi_{I}\right)=\sum_{J} \sum_{a_{1}, \ldots, a_{k}=1}^{m} \frac{\operatorname{sgn}\left(J J^{\prime}\right)}{k !} \cdot \operatorname{det}\left[x_{a_{p} j_{q}}\right] \operatorname{det}\left[\partial_{a_{p},-j_{q}^{\prime}}\right]
$$

where $J$ and $J^{\prime}$ are the same sets as in (4.3). We now obtain the second relation in (4.4) since both the determinants $\operatorname{det}\left[x_{a_{p} j_{q}}\right]$ and $\operatorname{det}\left[\partial_{a_{p},-j_{q}^{\prime}}\right]$ are skew-symmetric with respect to permutations of the sequence $\left(a_{1}, \ldots, a_{k}\right)$.

Now let us show that the right hand side of the first relation in (4.4) belongs to the ring of $O_{N}$-invariants in $\mathrm{U}\left(\mathfrak{s o}_{N}\right)$. Consider the element of $\Lambda^{2}\left(\mathbb{C}^{N}\right) \otimes \mathrm{U}\left(\mathfrak{s o}_{N}\right)$

$$
\Phi=\sum_{i<j}\left(e_{i} \wedge e_{j}\right) \otimes F_{i,-j}=\frac{1}{2} \cdot \sum_{i j}\left(e_{i} \wedge e_{j}\right) \otimes F_{i,-j}
$$


This element is invarant with respect to the diagonal action of the group $O_{N}$. That follows from the invariance of the element (3.1). By the definition (4.2) we have an equality in the algebra $\Lambda\left(\mathbb{C}^{N}\right) \otimes \mathrm{U}\left(\mathfrak{s o}_{N}\right)$

$$
\frac{\Phi^{k}}{k !}=\sum_{I}\left(e_{i_{1}} \wedge \ldots \wedge e_{i_{2 k}}\right) \otimes \Phi_{I}
$$

Extend the bilinear form $\langle$,$\rangle from \mathbb{C}^{N}$ to the exterior power $\Lambda^{2 k}\left(\mathbb{C}^{N}\right)$ by setting

$$
\left\langle e_{i_{1}} \wedge \ldots \wedge e_{i_{2 k}}, e_{-h_{2 k}} \wedge \ldots \wedge e_{-h_{1}}\right\rangle=\delta_{i_{1} h_{1}} \ldots \delta_{i_{2 k} h_{2 k}}
$$

if $h_{1}<\ldots<h_{2 k}$. This extension is also $O_{N}$-invarant. Extend it further to the $\mathbb{C}$-bilinear form on $\Lambda^{2 k}\left(\mathbb{C}^{N}\right) \otimes \mathrm{U}\left(\mathfrak{s o}_{N}\right)$ valued in $\mathrm{U}\left(\mathfrak{s o}_{N}\right)$

$$
\langle\xi \otimes X, \eta \otimes Y\rangle=\langle\xi, \eta\rangle \cdot X Y
$$

the latter form is $O_{N}$-equivariant. This implies $O_{N}$-invariance of the sum in (4.4)

$$
\sum_{I} \Phi_{I} \Phi_{I^{*}}=\left\langle\frac{\Phi^{k}}{k !}, \frac{\Phi^{k}}{k !}\right\rangle .
$$

Thus both sides of the first relation in (4.4) belong to $\mathrm{Z}\left(\mathfrak{s o}_{N}\right)$. We will show that they have the same eigenvalue in any irreducible $\mathfrak{s o}_{N}$-module $V_{\lambda}$. By definition, eigenvalue of the left hand side is the symmetric polynomial $(-1)^{k} e_{k}\left(l_{1}^{2}, \ldots, l_{n}^{2} \mid a\right)$ where we use the notation of Section 2 for $\mathfrak{g}=\mathfrak{s o}_{N}$. Let us now determine the eigenvalue of the right hand side. We will make use of the second equality in (4.4).

Suppose that $m \geqslant n$, then every $\mathfrak{s o}_{N}$-module $V_{\lambda}$ is contained in $\mathcal{P}$ as a submodule. Indeed, there is a well known formula for a $\mathfrak{s o}_{N}$-singular vector in $\mathcal{P}$ of weight $\lambda=\left(\lambda_{1}, \ldots, \lambda_{n}\right)$; see for instance [KV, Section 6$]$. For each $p=1, \ldots, n$ denote

$$
\Delta_{p}=\operatorname{det}\left[x_{a i}\right] ; \quad a=1, \ldots, p ; \quad i=-n, \ldots, p-1-n .
$$

One easily checks that the polynomial $v_{\lambda}=\Delta_{1}^{\lambda_{1}-\lambda_{2}} \ldots \Delta_{n-1}^{\lambda_{n-1}-\lambda_{n}} \Delta_{n}^{\lambda_{n}} \in \mathcal{P}$ satisfies

$$
\begin{array}{rlrl}
F_{i j} \cdot v_{\lambda} & =0, & i<j ; \\
F_{-p,-p} \cdot v_{\lambda}=\lambda_{n-p+1} v_{\lambda}, & 1 \leqslant p \leqslant n
\end{array}
$$

with respect to the action (4.1). Note that the polynomial $v_{\lambda}$ is of degree $|\lambda|$. So due to the second relation in (4.4) the right hand side of the first relation vanishes in every module $V_{\lambda}$ with $|\lambda|<k$; see (4.3). Take the image of the left hand side of (4.6) with respect to the Harish-Chandra isomorphism. Consider this image as a polynomial in $\lambda_{1}, \ldots, \lambda_{n}$. Due to Theorem 2.1 it now suffices to show that the leading component of this polynomial is $e_{k}\left(\lambda_{1}^{2}, \ldots, \lambda_{n}^{2}\right)$.

The leading terms of this polynomial arise from the summands of $\Phi_{I}$ and $\Phi_{I^{*}}$ which depend only on the generators $F_{i i}$. So here we may restrict the sum in (4.6) to the sets $I$ of the form $\left\{-p_{k}, \ldots,-p_{1}, p_{1}, \ldots, p_{k}\right\}$ where $1 \leqslant p_{1}<\ldots<p_{k} \leqslant n$. Then we get $I^{*}=I$. For any of these sets $I$ there are $2^{k} k !$ summands in (4.2) 
depending only on $F_{i i}$. All these summands are equal to each other. So the leading terms of our polynomial add up to

$$
\begin{gathered}
\sum_{1 \leqslant p_{1}<\ldots<p_{k} \leqslant n} \omega\left(F_{p_{1} p_{1}}^{2} \ldots F_{p_{k} p_{k}}^{2}\right)=\sum_{1 \leqslant p_{1}<\ldots<p_{k} \leqslant n} \lambda_{n-p_{1}+1}^{2} \ldots \lambda_{n-p_{k}+1}^{2} \\
=e_{k}\left(\lambda_{1}^{2}, \ldots, \lambda_{n}^{2}\right)
\end{gathered}
$$

The first equality in (4.4) is an alternative to Theorem 3.2 for $\mathfrak{g}=\mathfrak{s o}_{N}$. However, for $\mathfrak{g}=\mathfrak{s o}_{N}$ there is apparently no such alternative to Theorem 3.3. When $\mathfrak{g}=\mathfrak{s p}_{N}$ there is an analogue of Theorem 4.1, but only for the element $D_{k} \in \mathrm{Z}\left(\mathfrak{s p}_{N}\right)$ as opposed to the element $C_{k} \in \mathrm{Z}\left(\mathfrak{s p}_{N}\right)$; see Section 5 .

Corollary 4.2. Suppose that $N=2 n$. Then we have an equality in $\mathrm{U}\left(\mathfrak{s o}_{N}\right)$

$$
C_{n}=(-1)^{n} \cdot\left(\operatorname{Pf}\left[F_{i,-j}\right]\right)^{2} ; \quad i, j=-n, \ldots,-1,1, \ldots, n
$$

Let us now consider the operators from $\mathcal{P} \mathcal{D}$ which commute with the natural action of the group $O_{N}$ in $\mathcal{P}$. Description of this commutant is a particular case of the theory of reductive dual pairs due to R. Howe. Put $M=2 m$. Let $E_{ \pm a, \pm b}^{\prime}$ be the matrix units in End $\left(\mathbb{C}^{M}\right)$ corresponding to the standard basis of $e_{ \pm a}$ in $\mathbb{C}^{M}$. We will fix an embedding of the Lie algebra $\mathfrak{s p}_{M}$ into $\mathfrak{g l}_{M}$ similar to that used in Section 2 for $\mathfrak{s p}_{N}$. So the subalgebra $\mathfrak{s p}_{M} \subset \mathfrak{g l}_{M}$ will be spanned by the elements

$$
\begin{gathered}
F_{a b}^{\prime}=-F_{-a,-b}^{\prime}=E_{a b}^{\prime}-E_{-b,-a}^{\prime} \\
F_{a,-b}^{\prime}=E_{a,-b}^{\prime}+E_{b,-a}^{\prime}, \quad F_{-a, b}^{\prime}=E_{-a, b}^{\prime}+E_{-b, a}^{\prime} .
\end{gathered}
$$

We also fix the Cartan decomposition of $\mathfrak{s p}_{M}$ similar to that of $\mathfrak{s p}_{N}$. In particular, we will fix the Cartan subalgebra in $\mathfrak{s p}_{M}$ with the basis $\left(F_{-m,-m}^{\prime}, \ldots, F_{-1,-1}^{\prime}\right)$. Any weight $\lambda^{\prime}=\left(\lambda_{1}^{\prime}, \ldots, \lambda_{m}^{\prime}\right)$ of that Cartan subalgebra in $\mathfrak{s p}_{M}$ will be taken with respect to this basis. To distinguish the counterparts in $\mathrm{Z}\left(\mathfrak{s p}_{M}\right)$ of the elements $C_{1}, \ldots, C_{n} \in \mathrm{Z}\left(\mathfrak{s o}_{N}\right)$ we will denote them here by $C_{1}^{\prime}, \ldots, C_{m}^{\prime}$.

One can define an action of the Lie algebra $\mathfrak{s p}_{M}$ in the space $\mathcal{P}$ by

$$
\begin{gathered}
F_{a b}^{\prime} \mapsto \sum_{i} x_{a i} \partial_{b i}+\frac{N}{2} \cdot \delta_{a b} \\
F_{a,-b}^{\prime} \mapsto-\sum_{i} x_{a i} x_{b,-i}, \quad F_{-a, b}^{\prime} \mapsto \sum_{i} \partial_{a i} \partial_{b,-i}
\end{gathered}
$$

Denote by $\gamma^{\prime}$ the corresponding representation $\mathrm{U}\left(\mathfrak{s p}_{M}\right) \rightarrow \mathcal{P} \mathcal{D}$. The image of this representation coincides with the commutant in $\mathcal{P D}$ of the action of the group $O_{N}$ in $\mathcal{P}[\mathrm{H}$, Theorem 8$]$. In particular, we have the equality in $\mathcal{P} \mathcal{D}$

$$
\gamma\left(\mathrm{Z}\left(\mathfrak{s o}_{N}\right)\right)=\gamma^{\prime}\left(\mathrm{Z}\left(\mathfrak{s p}_{M}\right)\right)
$$

We will describe the correspondence between the generators $C_{1}, \ldots, C_{n} \in \mathrm{Z}\left(\mathfrak{s o}_{N}\right)$ and $C_{1}^{\prime}, \ldots, C_{m}^{\prime} \in \mathrm{Z}\left(\mathfrak{s p}_{M}\right)$ implied by $(4.7)$; cf. [HU, Section 1]. 
As in Section 3 let $u$ be a complex variable. Introduce the generating functions

$$
\begin{aligned}
C(u) & =1+\sum_{k=1}^{n} \frac{C_{k}}{\left(u^{2}-\left(\frac{N}{2}-1\right)^{2}\right) \ldots\left(u^{2}-\left(\frac{N}{2}-k\right)^{2}\right)} \in \mathrm{Z}\left(\mathfrak{s o}_{N}\right)(u), \\
C^{\prime}(u) & =1+\sum_{k=1}^{m} \frac{C_{k}^{\prime}}{\left(u^{2}-m^{2}\right) \ldots\left(u^{2}-(m-k+1)^{2}\right)} \in \mathrm{Z}\left(\mathfrak{s p}_{M}\right)(u) .
\end{aligned}
$$

Also put in $\mathbb{C}(u)$

$$
\alpha(u)=\prod_{a=1}^{m} \frac{u^{2}-\left(\frac{N}{2}-a\right)^{2}}{u^{2}-a^{2}} .
$$

Relations between the images of $C_{1}, \ldots, C_{n} \in \mathrm{Z}\left(\mathfrak{s o}_{N}\right)$ and $C_{1}^{\prime}, \ldots, C_{m}^{\prime} \in \mathrm{Z}\left(\mathfrak{s p}_{M}\right)$ in $\mathcal{P D}$ respectively under $\gamma$ and $\gamma^{\prime}$ are described by the following proposition.

Proposition 4.3. We have an equality in $\mathcal{P} \mathcal{D}(u)$

$$
\alpha(u) \cdot \gamma(C(u))=\gamma^{\prime}\left(C^{\prime}(u)\right)
$$

Proof. The half-sum of positive roots for $\mathfrak{s p}_{M}$ is $\rho^{\prime}=(m, \ldots, 1)$. Let $l_{a}^{\prime}=\lambda_{a}^{\prime}+\rho_{a}^{\prime}$ be the analogues for $\mathfrak{s p}_{M}$ of the variables $l_{p}=\lambda_{p}+\rho_{p}$ for $\mathfrak{s o}_{N}$. Recall that here we have

$$
\rho=\left(\frac{N}{2}-1, \ldots,\left\{\frac{N}{2}\right\}\right)
$$

where the braces mean the fractional part. The images of $C(u)$ and $C^{\prime}(u)$ under the relevant Harish-Chandra isomorphisms are equal to

$$
\frac{\left(u^{2}-l_{1}^{2}\right) \ldots\left(u^{2}-l_{n}^{2}\right)}{\left(u^{2}-\rho_{1}^{2}\right) \ldots\left(u^{2}-\rho_{n}^{2}\right)} \quad \text { and } \quad \frac{\left(u^{2}-l_{1}^{\prime 2}\right) \ldots\left(u^{2}-l_{m}^{\prime 2}\right)}{\left(u^{2}-\rho_{1}^{\prime 2}\right) \ldots\left(u^{2}-\rho_{m}^{\prime 2}\right)}
$$

respectively. By the definition of the elements $C_{1}, \ldots, C_{n}$ the first equality follows from the identity $(2.8)$ applied to $t=u^{2}$ and $\left(z_{1}, \ldots, z_{n}\right)=\left(l_{1}^{2}, \ldots, l_{n}^{2}\right)$; see also (2.12) and (2.13). The second equality is obtained in a similar way.

Let us now employ the decomposition [KV, Theorem 7.2] of the space $\mathcal{P} \mathcal{D}$ into a direct sum of irreducible $O_{N} \times \mathfrak{s p}_{M}$-modules. By comparing the two rational functions in (4.10) one can now check that in each of these irreducible modules the eigenvalues of $\alpha(u) \cdot C(u) \in \mathrm{Z}\left(\mathfrak{s o}_{N}\right)(u)$ and $C^{\prime}(u) \in \mathrm{Z}\left(\mathfrak{s p}_{M}\right)(u)$ coincide. This is a straightforward calculation, and we refer to $[\mathrm{KV}$, Section 6] for details

By using the equality (4.9) each of the images $\gamma^{\prime}\left(C_{1}^{\prime}\right), \ldots, \gamma^{\prime}\left(C_{m}^{\prime}\right)$ in $\mathcal{P} \mathcal{D}$ can be expressed as a linear combination of the operators $\gamma\left(C_{1}\right), \ldots, \gamma\left(C_{n}\right)$; see the next theorem. But for the latter operators we have already obtained the explicit formulas (4.4). Recall that for $k>n$ we have $C_{k}=0$ by definition. Put $C_{0}=1$.

Theorem 4.4. Denote $d=m-\frac{N}{2}+1$. Then for any $k=1, \ldots, m$ we have

$$
\gamma^{\prime}\left(C_{k}^{\prime}\right)=\sum_{l=0}^{k} \gamma\left(C_{l}\right) \cdot \prod_{s=l}^{k-1} \frac{(m-s)\left(\frac{N}{2}-s\right)(d+l-s)}{(k-s)}
$$

Proof. We will check that (4.11) solves the equation (4.9) for $\gamma^{\prime}\left(C_{1}^{\prime}\right), \ldots, \gamma^{\prime}\left(C_{m}^{\prime}\right)$. Let us subsitute (4.11) into (4.9) using the definitions of the generating functions 
$C(u)$ and $C^{\prime}(u)$. Denote by $f_{k l}$ the product over $s=l, \ldots, k-1$ at the right hand side of (4.11). Then we have to check for any fixed $l=0,1, \ldots, \min (m, n)$ the equality

$$
\prod_{r=1}^{m-l}\left(u^{2}-(r-d)^{2}\right)=\sum_{k=l}^{m} f_{k l} \cdot \prod_{r=1}^{m-k}\left(u^{2}-r^{2}\right) .
$$

Actually, the latter equality is valid for any $l=0,1, \ldots, m$. This can be easily verified by induction on $m-l=0,1, \ldots$

Observe that if $N=2 n$ and $m>n$ then the coefficient $f_{k l}$ vanishes for any $k>n$. Therefore we have the following corollary to Theorem 4.4.

Corollary 4.5. Suppose $N=2 n$ and $m>n$. Then $\gamma^{\prime}\left(C_{n+1}^{\prime}\right), \ldots, \gamma^{\prime}\left(C_{m}^{\prime}\right)=0$.

Also observe that if $N=2 n$ and $m \geqslant n-1$ then $f_{k l}=0$ for $k>m-n+1+l$. In particular, we have another corollary; it also follows from Proposition 4.3 directly.

Corollary 4.6. Suppose that $N=2 n$ and $m=n-1$. Then $\gamma^{\prime}\left(C_{k}^{\prime}\right)=\gamma\left(C_{k}\right)$ for any $k=1, \ldots, m$.

Note that due to Proposition 2.3 we have an equality in $\mathrm{Z}\left(\mathfrak{s o}_{N}\right)\left[\left[u^{-1}\right]\right]$

$$
1+\sum_{k=1}^{\infty} \frac{D_{k}}{\left(u^{2}-\left(\frac{N}{2}\right)^{2}\right) \ldots\left(u^{2}-\left(\frac{N}{2}+k-1\right)^{2}\right)}=C(u)^{-1}
$$

Thus the series $C(u)^{-1}$ can be regarded as a generating function for the elements $D_{1}, D_{2}, \ldots \in \mathrm{Z}\left(\mathfrak{s o}_{N}\right)$; cf. the equality (5.11) for the Lie algebra $\mathfrak{g}=\mathfrak{s p}_{N}$ below.

Results of this section rely on the fact that real Lie groups $O_{N}(\mathbb{R})$ and $S p_{M}(\mathbb{R})$ form a reductive dual pair inside $S p_{M N}(\mathbb{R})$; see $[\mathrm{EHW}$. The case of another dual pair of $S p_{N}(\mathbb{R})$ and $O_{M}^{*}(\mathbb{R})$ in $S p_{M N}(\mathbb{R})$ will be considered in the next section.

\section{Capelli identity for the Lie algebra $\mathfrak{s p}_{N}$}

In this section we will keep fixed two positive integers $m$ and $N$. We will consider a natural representation of the enveloping algebra $\mathrm{U}\left(\mathfrak{s p}_{N}\right)$ by differential operators with polynomial coefficients on the space $\mathbb{C}^{m} \otimes \mathbb{C}^{N}$. Results of this section will be similar to those of Section 4 . But here for any $k=1,2, \ldots$ we will give an explicit formula for the differential operator corresponding to the element $D_{k} \in \mathrm{Z}\left(\mathfrak{s p}_{N}\right)$ rather than $C_{k} \in \mathrm{Z}\left(\mathfrak{s p}_{N}\right)$. This formula may be still regarded as an analogue for $\mathfrak{s p}_{N}$ of the Capelli identity [C] for the Lie algebra $\mathfrak{g l}_{N} ;$ cf. [N1, Example 2].

Put $N=2 n$. Let the indices $i, j$ run through the set $\{-n, \ldots,-1,1, \ldots, n\}$. The indices $a, b$ will again run through the set $\{1, \ldots, m\}$. Let $\mathcal{P}$ be the same ring of polynomials in the variableas $x_{a i}$ as introduced in the previous section. We will use the embedding of the group $S p_{N}$ into $G L_{N}$ chosen in Section 2. The restriction to $\mathfrak{s p}_{N}$ of the action (1.2) of $\mathfrak{g l}_{N}$ in $\mathcal{P}$ then takes the form

$$
F_{i j} \mapsto \sum_{a}\left(x_{a i} \partial_{a j}-\operatorname{sgn}(i j) \cdot x_{a,-j} \partial_{a,-i}\right)
$$

Thus we get a representation $\mathrm{U}\left(\mathfrak{s p}_{N}\right) \rightarrow \mathcal{P} \mathcal{D}$ which we will again denote by $\gamma$. For each $k=1,2, \ldots$ we will give an explicit formula for the operator $\gamma\left(D_{k}\right) \in \mathcal{P} \mathcal{D}$. 
Put $\tilde{F}_{i j}=\operatorname{sgn}(i) \cdot F_{i j}$. Then by definition we have $\tilde{F}_{i,-j}=\tilde{F}_{j,-i}$. Let $I$ be any sequence of indices $i$ with $2 k$ elements. Suppose these elements are $i_{1} \leqslant \ldots \leqslant i_{2 k}$. Take an element of the algebra $\mathrm{U}\left(\mathfrak{s p}_{N}\right)$

$$
\Psi_{I}=\sum_{\sigma} \frac{1}{2^{k} k !} \cdot \tilde{F}_{i_{\sigma(1)},-i_{\sigma(2)}} \ldots \tilde{F}_{i_{\sigma(2 k-1)},-i_{\sigma(2 k)}}
$$

where $\sigma$ runs through the symmetric group $S_{2 k}$. The element (5.2) may be called the Hafnian of the symmetric matrix $\left[\tilde{F}_{i_{p},-i_{q}}\right]$ with the indices $p, q=1, \ldots, 2 k$. This term was devised by E. Caianiello for a symmetric matrix with commuting entries; see [K, Section 2]. The factors of the monomial in the sum (5.2) do not commute in general. Still we will use this term and write

$$
\Psi_{I}=\operatorname{Hf}\left[\tilde{F}_{i_{p},-i_{q}}\right] ; \quad p, q=1, \ldots, 2 k .
$$

Let us denote $I^{*}=\left(-i_{2 k}, \ldots,-i_{1}\right)$. Further, let $A$ be any collection of indices $a$ with $k$ elements. Suppose these elements are $a_{1}, \ldots, a_{k}$. Denote in $\mathcal{P} \mathcal{D}$

$$
\Theta_{A I}=\sum_{J} \operatorname{sgn}\left(j_{1} \ldots j_{k}\right) \cdot \operatorname{per}\left[x_{a_{p} j_{q}}\right] \operatorname{per}\left[\partial_{a_{p},-j_{q}^{\prime}}\right]
$$

where the sum is taken over all partitions of $I$ into subsequences $J=\left(j_{1}, \ldots, j_{k}\right)$ and $J^{\prime}=\left(j_{1}^{\prime}, \ldots, j_{k}^{\prime}\right)$ each of length $k$. So $j_{1} \leqslant \ldots \leqslant j_{k}$ and $j_{1}^{\prime} \leqslant \ldots \leqslant j_{k}^{\prime}$. Here the permanents are taken with respect to the indices $p, q=1, \ldots, k$. Let $f_{ \pm 1}, \ldots, f_{ \pm n}$ be the multiplicities of the numbers $\pm 1, \ldots, \pm n$ in $I$ respectively. Let $d_{1}, \ldots, d_{m}$ be the multiplicities of $1, \ldots, m$ in $A$. The next statement is an analogue of Theorem 4.1, it provides an explicit formula for $\gamma\left(D_{k}\right) \in \mathcal{P} \mathcal{D}$.

Theorem 5.1. We have the equalities respectively in $\mathrm{U}\left(\mathfrak{s p}_{N}\right)$ and $\mathcal{P} \mathcal{D}$

$$
D_{k}=(-1)^{k} \cdot \sum_{I} \frac{\operatorname{sgn}\left(i_{1} \ldots i_{2 k}\right) \cdot \Psi_{I} \Psi_{I^{*}}}{f_{1} ! f_{-1} ! \ldots f_{n} ! f_{-n} !} \quad \text { and } \gamma\left(\Psi_{I}\right)=\sum_{A} \frac{\Theta_{A I}}{d_{1} ! \ldots d_{m} !} \text {. }
$$

Proof. We start with verifying the second equality in (5.4). By definition $\Psi_{I}$ equals

$$
\begin{gathered}
\frac{1}{2^{k} k !} \cdot \sum_{\sigma}\left(\operatorname{sgn}\left(i_{\sigma(1)}\right) \cdot E_{i_{\sigma(1)},-i_{\sigma(2)}}+\operatorname{sgn}\left(i_{\sigma(2)}\right) \cdot E_{i_{\sigma(2)},-i_{\sigma(1)}}\right) \times \ldots \times \\
\left(\operatorname{sgn}\left(i_{\sigma(2 k-1)}\right) \cdot E_{i_{\sigma(2 k-1)},-i_{\sigma(2 k)}}+\operatorname{sgn}\left(i_{\sigma(2 k)}\right) \cdot E_{i_{\sigma(2 k)},-i_{\sigma(2 k-1)}}\right)= \\
\frac{1}{k !} \cdot \sum_{\sigma} \operatorname{sgn}\left(i_{\sigma(1)} i_{\sigma(3)} \ldots i_{\sigma(2 k-1)}\right) \cdot E_{i_{\sigma(1)},-i_{\sigma(2)}} \ldots E_{i_{\sigma(2 k-1)},-i_{\sigma(2 k)}}
\end{gathered}
$$

in $\mathrm{U}\left(\mathfrak{s p}_{N}\right) \subset \mathrm{U}\left(\mathfrak{g l}_{N}\right)$. Therefore the image $\gamma\left(\Psi_{I}\right) \in \mathcal{P} \mathcal{D}$ equals the sum

$$
\sum_{\sigma} \sum_{a_{1}, \ldots, a_{k}=1}^{m} \frac{\operatorname{sgn}\left(i_{\sigma(1)} \ldots i_{\sigma(2 k-1)}\right)}{k !} \cdot x_{a_{1} i_{\sigma(1)}} \partial_{a_{1},-i_{\sigma(2)}} \ldots x_{a_{k} i_{\sigma(2 k-1)}} \partial_{a_{k},-i_{\sigma(2 k)}} .
$$

Move to the right each operator $\partial_{a_{p},-i_{\sigma(2 p)}}$ in every monomial above, commuting it consecutively with the multiplication operators $x_{a_{q} i_{\sigma(2 q-1)}}$ for $q=p+1, \ldots, k$. Let us start with the operator $\partial_{a_{1},-i_{\sigma(2)}}$. Take the commutator

$$
\left[\partial_{a_{1},-i_{\sigma(2)}}, x_{a_{2} i_{\sigma(3)}}\right]=\delta_{a_{1} a_{2}} \delta_{-i_{\sigma(2)}, i_{\sigma(3)}}
$$


The above sum over $\sigma$ contains two such commutators with opposite signs. Indeed, if $\bar{\sigma} \in S_{2 k}$ is such that $\bar{\sigma}(r)=\sigma(r)$ for $r \neq 2,3$ and $\bar{\sigma}(2)=\sigma(3), \bar{\sigma}(3)=\sigma(2)$ then

$$
\left[\partial_{a_{1},-i_{\bar{\sigma}(2)}}, x_{a_{2} i_{\bar{\sigma}(3)}}\right]=\left[\partial_{a_{1},-i_{\sigma(2)}}, x_{a_{2} i_{\sigma(3)}}\right]
$$

whilst

$$
\operatorname{sgn}\left(i_{\bar{\sigma}(1)} i_{\bar{\sigma}(3)} \ldots i_{\bar{\sigma}(2 k-1)}\right)=-\operatorname{sgn}\left(i_{\sigma(1)} i_{\sigma(3)} \ldots i_{\sigma(2 k-1)}\right)
$$

for $i_{\sigma(2)}=-i_{\sigma(3)}$. So by repeating this argument we bring $\gamma\left(\Psi_{I}\right)$ to the form

$$
\sum_{\sigma} \sum_{a_{1}, \ldots, a_{k}=1}^{m} \frac{\operatorname{sgn}\left(i_{\sigma(1)} \ldots i_{\sigma(2 k-1)}\right)}{k !} \cdot x_{a_{1} i_{\sigma(1)}} \ldots x_{a_{k} i_{\sigma(2 k-1)}} \partial_{a_{1},-i_{\sigma(2)}} \ldots \partial_{a_{k},-i_{\sigma(2 k)}}
$$

The latter sum in $\mathcal{P} \mathcal{D}$ can be obviously rewritten as

$$
\gamma\left(\Psi_{I}\right)=\sum_{J} \sum_{a_{1}, \ldots, a_{k}=1}^{m} \frac{\operatorname{sgn}\left(j_{1} \ldots j_{k}\right)}{k !} \cdot \operatorname{per}\left[x_{a_{p} j_{q}}\right] \operatorname{per}\left[\partial_{a_{p},-j_{q}^{\prime}}\right]
$$

where $J$ and $J^{\prime}$ are the same as in (5.3). We now obtain the second relation in (5.4) since both the permanents per $\left[x_{a_{p} j_{q}}\right]$ and per $\left[\partial_{a_{p},-j_{q}^{\prime}}\right]$ are symmetric with respect to permutations of the sequence $\left(a_{1}, \ldots, a_{k}\right)$.

Now let us show that the right hand side of the first relation in (5.4) belongs to the centre of $\mathrm{U}\left(\mathfrak{s p}_{N}\right)$. Consider the element of $\mathrm{S}^{2}\left(\mathbb{C}^{N}\right) \otimes \mathrm{U}\left(\mathfrak{s p}_{N}\right)$

$$
\Psi=\frac{1}{2} \cdot \sum_{i j} e_{i} e_{j} \otimes \tilde{F}_{i,-j}
$$

This element is invarant with respect to the diagonal action of the group $S p_{N}$. That again follows from the invariance of the element (3.1). By the definition (5.2) we have an equality in the algebra $\mathrm{S}\left(\mathbb{C}^{N}\right) \otimes \mathrm{U}\left(\mathfrak{s o}_{N}\right)$

$$
\frac{\Psi^{k}}{k !}=\sum_{I} \frac{e_{i_{1}} \ldots e_{i_{2 k}}}{f_{1} ! f_{-1} ! \ldots f_{n} ! f_{-n} !} \otimes \Psi_{I}
$$

Extend the bilinear form $\langle$,$\rangle from \mathbb{C}^{N}$ to the symmetric power $\mathrm{S}^{2 k}\left(\mathbb{C}^{N}\right)$ by

$$
\left\langle e_{i_{1}} \ldots e_{i_{2 k}}, e_{-h_{2 k}} \ldots e_{-h_{1}}\right\rangle=\delta_{i_{1} h_{1}} \ldots \delta_{i_{2 k} h_{2 k}} \cdot f_{1} ! f_{-1} ! \ldots f_{n} ! f_{-n} ! \cdot \operatorname{sgn}\left(i_{1} \ldots i_{2 k}\right)
$$

This extension is also $S p_{N}$-invarant. Extend it further to the $\mathbb{C}$-bilinear form on $\mathrm{S}^{2 k}\left(\mathbb{C}^{N}\right) \otimes \mathrm{U}\left(\mathfrak{s p}_{N}\right)$ valued in $\mathrm{U}\left(\mathfrak{s p}_{N}\right)$

$$
\langle\xi \otimes X, \eta \otimes Y\rangle=\langle\xi, \eta\rangle \cdot X Y
$$

the latter form is $S p_{N}$-equivariant. It implies $S p_{N}$-invariance of the sum in $(5.4)$

$$
\sum_{I} \frac{\operatorname{sgn}\left(i_{1} \ldots i_{2 k}\right) \cdot \Psi_{I} \Psi_{I^{*}}}{f_{1} ! f_{-1} ! \ldots f_{n} ! f_{-n} !}=\left\langle\frac{\Psi^{k}}{k !}, \frac{\Psi^{k}}{k !}\right\rangle
$$


Thus both sides of the first relation in (5.4) belong to $\mathrm{Z}\left(\mathfrak{s p}_{N}\right)$. We will show that they have the same eigenvalue in any irreducible $\mathfrak{s p}_{N}$-module $V_{\lambda}$. By definition, the eigenvalue of the left hand side is the symmetric polynomial $h_{k}\left(l_{1}^{2}, \ldots, l_{n}^{2} \mid a\right)$ where we use the notation of Section 2 for $\mathfrak{g}=\mathfrak{s p}_{N}$. Let us now determine the eigenvalue of the right hand side. We will make use of the second equality in (5.4).

Suppose that $m \geqslant n$, then every $\mathfrak{s p}_{N}$-module $V_{\lambda}$ is contained in $\mathcal{P}$ as a submodule. Indeed, the vector $v_{\lambda} \in \mathcal{P}$ defined in the proof of Theorem 4.1 is also singular of weight $\lambda=\left(\lambda_{1}, \ldots, \lambda_{n}\right)$ with respect to the action (5.1) of $\mathfrak{s p}_{N}$. That is,

$$
\begin{aligned}
F_{i j} \cdot v_{\lambda} & =0, & i<j ; \\
F_{-p,-p} \cdot v_{\lambda} & =\lambda_{n-p+1} v_{\lambda}, & 1 \leqslant p \leqslant n
\end{aligned}
$$

again. The polynomial $v_{\lambda}$ is of degree $|\lambda|$. By second relation in (5.4) the right hand side of the first relation vanishes in every module $V_{\lambda}$ with $|\lambda|<k$; see (5.3).

Take the image of the left hand side of (5.5) with respect to the Harish-Chandra isomorphism. Let us consider this image as a polynomial in $\lambda_{1}, \ldots, \lambda_{n}$. Due to Theorem 2.1 it now suffices to show that the leading component of this polynomial is $(-1)^{k} \cdot h_{k}\left(\lambda_{1}^{2}, \ldots, \lambda_{n}^{2}\right)$.

The leading terms of this polynomial arise from the summands of $\Psi_{I}$ and $\Psi_{I^{*}}$ which depend only on the generators $F_{i i} \in \mathfrak{s p}_{N}$. So here we may restrict the sum in (5.5) to the sequences $I$ of the form $\left\{-p_{k}, \ldots,-p_{1}, p_{1}, \ldots, p_{k}\right\}$ where $1 \leqslant p_{1} \leqslant \ldots \leqslant p_{k} \leqslant n$. Then we get $I^{*}=I$. For any of these sequences $I$ there are $2^{k} k ! \cdot f_{1} ! \ldots f_{n}$ ! summands in (5.2) depending only on $F_{i i}$. All these summands are equal to each other. So the leading terms of our polynomial add up to

$$
\begin{gathered}
\sum_{1 \leqslant p_{1} \leqslant \ldots \leqslant p_{k} \leqslant n} \omega\left(F_{p_{1} p_{1}}^{2} \ldots F_{p_{k} p_{k}}^{2}\right) \cdot(-1)^{k}=\sum_{1 \leqslant p_{1} \leqslant \ldots \leqslant p_{k} \leqslant n} \lambda_{n-p_{1}+1}^{2} \ldots \lambda_{n-p_{k}+1}^{2} \cdot(-1)^{k} \\
=(-1)^{k} h_{k}\left(\lambda_{1}^{2}, \ldots, \lambda_{n}^{2}\right) \square
\end{gathered}
$$

The first equality in (5.4) is an alternative to Theorem 3.3 for $\mathfrak{g}=\mathfrak{s p}_{N}$. However, when $\mathfrak{g}=\mathfrak{s p}_{N}$ there is apparently no such alternative to Theorem 3.2.

Let us now consider the operators from $\mathcal{P} \mathcal{D}$ which commute with the action (5.1) of the Lie algebra $\mathfrak{s p}_{N}$ in $\mathcal{P}$. Description of this commutant is another particular case of the theory of reductive dual pairs $[\mathrm{H}]$. Put $M=2 m$. As in Section 4, denote by $E_{ \pm a, \pm b}^{\prime}$ the matrix units in $\operatorname{End}\left(\mathbb{C}^{M}\right)$ corresponding to the standard basis of $e_{ \pm a}$ in $\mathbb{C}^{M}$. Fix an embedding of the Lie algebra $\mathfrak{s o}_{M}$ into $\mathfrak{g l}_{M}$ similar to that used in Section 2 for $\mathfrak{s o}_{N}$. So the subalgebra $\mathfrak{s o}_{M} \subset \mathfrak{g l}_{M}$ will be spanned by

$$
\begin{gathered}
F_{a b}^{\prime}=-F_{-a,-b}^{\prime}=E_{a b}^{\prime}-E_{-b,-a}^{\prime}, \\
F_{a,-b}^{\prime}=E_{a,-b}^{\prime}-E_{b,-a}^{\prime}, \quad F_{-a, b}^{\prime}=E_{-a, b}^{\prime}-E_{-b, a}^{\prime} .
\end{gathered}
$$

We also fix the Cartan decomposition of $\mathfrak{s o}_{M}$ similar to that of $\mathfrak{s o}_{N}$. In particular, we will fix the Cartan subalgebra in $\mathfrak{s o}_{M}$ with the basis $\left(F_{-m,-m}^{\prime}, \ldots, F_{-1,-1}^{\prime}\right)$. Any weight $\lambda^{\prime}=\left(\lambda_{1}^{\prime}, \ldots, \lambda_{m}^{\prime}\right)$ of that Cartan subalgebra in $\mathfrak{s o}_{M}$ will be taken with respect to this basis. To distinguish the counterparts in $\mathrm{Z}\left(\mathfrak{s o}_{M}\right)$ of the elements $D_{1}, D_{2}, \ldots \in \mathrm{Z}\left(\mathfrak{s p}_{N}\right)$ we will denote them by $D_{1}^{\prime}, D_{2}^{\prime}, \ldots$ in this section. 
One can define an action of the Lie algebra $\mathfrak{s o}_{M}$ in the space $\mathcal{P}$ by

$$
\begin{gathered}
F_{a b}^{\prime} \mapsto \sum_{i} x_{a i} \partial_{b i}+n \cdot \delta_{a b} \\
F_{a,-b}^{\prime} \mapsto \sum_{i} \operatorname{sgn}(i) \cdot x_{a i} x_{b,-i}, \quad F_{-a, b}^{\prime} \mapsto \sum_{i} \operatorname{sgn}(i) \cdot \partial_{a i} \partial_{b,-i}
\end{gathered}
$$

Denote by $\gamma^{\prime}$ the corresponding representation $\mathrm{U}\left(\mathfrak{s o}_{M}\right) \rightarrow \mathcal{P D}$. The image of this representation coincides with the commutant in $\mathcal{P D}$ of the action of the Lie algebra $\mathfrak{s p}_{N}$ in $\mathcal{P}[\mathrm{H}$, Theorem 8]. In particular, we have the equality in $\mathcal{P} \mathcal{D}$

$$
\gamma\left(\mathrm{Z}\left(\mathfrak{s p}_{N}\right)\right)=\gamma^{\prime}\left(\mathrm{Z}\left(\mathfrak{s o}_{M}\right)\right)
$$

We will describe the correspondence between the generators $D_{1}, D_{2}, \ldots \in \mathrm{Z}\left(\mathfrak{s p}_{N}\right)$ and $D_{1}^{\prime}, D_{2}^{\prime}, \ldots \in \mathrm{Z}\left(\mathfrak{s o}_{M}\right)$ implied by $(5.6)$.

As in Section 4 let $u$ be a complex variable. Introduce the generating functions

$$
\begin{aligned}
D(u) & =1+\sum_{k=1}^{\infty} \frac{D_{k}}{\left(u^{2}-(n+1)^{2}\right) \ldots\left(u^{2}-(n+k)^{2}\right)} \in \mathrm{Z}\left(\mathfrak{s p}_{N}\right)\left[\left[u^{-1}\right]\right], \\
D^{\prime}(u) & =1+\sum_{k=1}^{\infty} \frac{D_{k}^{\prime}}{\left(u^{2}-m^{2}\right) \ldots\left(u^{2}-(m+k-1)^{2}\right)} \in \mathrm{Z}\left(\mathfrak{s o}_{M}\right)\left[\left[u^{-1}\right]\right] .
\end{aligned}
$$

Also put in $\mathbb{C}(u)$

$$
\beta(u)=\prod_{a=1}^{m} \frac{u^{2}-(a-1)^{2}}{u^{2}-(n-a+1)^{2}} .
$$

Relations between the images of $D_{1}, D_{2}, \ldots \in \mathrm{Z}\left(\mathfrak{s p}_{N}\right)$ and $D_{1}^{\prime}, D_{2}^{\prime}, \ldots \in \mathrm{Z}\left(\mathfrak{s o}_{M}\right)$ in $\mathcal{P} \mathcal{D}$ respectively under $\gamma$ and $\gamma^{\prime}$ can be described as follows.

Proposition 5.2. We have an equality in $\mathcal{P} \mathcal{D}(u)$

$$
\beta(u) \cdot \gamma(D(u))=\gamma^{\prime}\left(D^{\prime}(u)\right)
$$

Proof. It will be similar to that of Proposition 4.3. The half-sum of positive roots for $\mathfrak{s o}_{M}$ is $\rho^{\prime}=(m-1, \ldots, 0)$. Let $l_{a}^{\prime}=\lambda_{a}^{\prime}+\rho_{a}^{\prime}$ be the analogues for $\mathfrak{s o}_{M}$ of the variables $l_{p}=\lambda_{p}+\rho_{p}$ for $\mathfrak{s p}_{N}$. Recall that here we have $\rho=(n, \ldots, 1)$. The images of $D(u)$ and $D^{\prime}(u)$ under the relevant Harish-Chandra isomorphisms are

$$
\frac{\left(u^{2}-\rho_{1}^{2}\right) \ldots\left(u^{2}-\rho_{n}^{2}\right)}{\left(u^{2}-l_{1}^{2}\right) \ldots\left(u^{2}-l_{n}^{2}\right)} \quad \text { and } \quad \frac{\left(u^{2}-\rho_{1}^{\prime 2}\right) \ldots\left(u^{2}-\rho_{m}^{\prime 2}\right)}{\left(u^{2}-l_{1}^{\prime 2}\right) \ldots\left(u^{2}-l_{m}^{\prime 2}\right)}
$$

respectively. By the definitions of $D_{1}, D_{2}, \ldots$ here the first equality follows from (2.9) applied to $t=u^{2}$ and $\left(z_{1}, \ldots, z_{n}\right)=\left(l_{1}^{2}, \ldots, l_{n}^{2}\right)$; see also (2.12) and (2.13). The second equality is obtained in a similar way.

Let us now employ the decomposition [EHW, Section 9] of the space $\mathcal{P} \mathcal{D}$ into a direct sum of irreducible $\mathfrak{s p}_{N} \times \mathfrak{s o}_{M}$-modules. By comparing the two rational functions in (5.8) one can now check that in each of these irreducible modules the eigenvalues of $\beta(u) \cdot D(u) \in \mathrm{Z}\left(\mathfrak{s p}_{N}\right)(u)$ and $D^{\prime}(u) \in \mathrm{Z}\left(\mathfrak{s o}_{M}\right)(u)$ coincide. This is again a straightforward calculation, and we will omit the details

By using the equality (5.7) each of the images $\gamma^{\prime}\left(D_{1}^{\prime}\right), \gamma^{\prime}\left(D_{2}^{\prime}\right), \ldots$ in $\mathcal{P} \mathcal{D}$ can be expressed as a linear combination of the operators $\gamma\left(D_{1}\right), \gamma\left(D_{2}\right), \ldots$; see the next theorem. For the latter operators we already have the formulas (5.4). Put $D_{0}=1$. 
Theorem 5.3. Put $d=n-m+1$. Then for any $k=1,2, \ldots$ we have

$$
\gamma^{\prime}\left(D_{k}^{\prime}\right)=\sum_{l=0}^{k} \gamma\left(D_{l}\right) \cdot \prod_{s=l}^{k-1} \frac{(m+s)(n+s)(d-k+s+1)}{(s-l+1)} .
$$

Proof. By Proposition 5.2 and the definitions of $D(u), D^{\prime}(u)$ the differential operator $\gamma^{\prime}\left(D_{k}^{\prime}\right)$ is a linear combination of $\gamma\left(D_{1}\right), \ldots, \gamma\left(D_{k}\right)$ where the coefficients are certain rational functions in $m, n$. Therefore it suffices to prove (5.9) only for $d \geqslant 0$. Suppose this is the case. We will check that (5.9) solves the equation (5.7) for $\gamma^{\prime}\left(D_{1}^{\prime}\right), \gamma^{\prime}\left(D_{2}^{\prime}\right), \ldots$. Let us substitute (5.9) into (5.7) using the definitions of the generating functions $D(u)$ and $D^{\prime}(u)$. Denote by $g_{k l}$ the product over $s=l, \ldots, k-1$ at the right hand side of (5.9). Observe that $g_{k l}=0$ for any $k>d+l$. So we have to check for any fixed $l=0,1,2, \ldots$ the equality in $\mathbb{C}(u)$

$$
\prod_{r=0}^{m+l-1} \frac{1}{u^{2}-(r+d)^{2}}=\sum_{k=l}^{l+d} g_{k l} \cdot \prod_{r=0}^{m+k-1} \frac{1}{u^{2}-r^{2}}
$$

Actually, the latter equality is valid for any $l=-m, 1-m, \ldots$. This can be easily verified by induction on $m+l=0,1, \ldots$

We also have a direct corollary to Proposition 5.2, it is an analogue of Corollary 4.6.

Corollary 5.4. Suppose $n=m-1$. Then $\gamma^{\prime}\left(D_{k}^{\prime}\right)=\gamma\left(D_{k}\right)$ for any $k=1,2, \ldots$

Note that due to Proposition 2.3 we have an equality in $\mathrm{Z}\left(\mathfrak{s p}_{N}\right)\left[\left[u^{-1}\right]\right]$

$$
1+\sum_{k=1}^{n} \frac{C_{k}}{\left(u^{2}-n^{2}\right) \ldots\left(u^{2}-(n-k+1)^{2}\right)}=D(u)^{-1}
$$

Similarly to (4.8), denote this generating function by $C(u)$. Then by definition we have the equality in $\mathrm{Z}\left(\mathfrak{s p}_{N}\right)\left[\left[u^{-1}\right]\right]$

$$
1+\sum_{k=1}^{\infty} \frac{D_{k}}{\left(u^{2}-(n+1)^{2}\right) \ldots\left(u^{2}-(n+k)^{2}\right)}=C(u)^{-1} .
$$

In the next section we will give another formula for the generating function $C(u)$.

\section{Quantum determinant of the element $F(u)$}

Here we will give an explicit formula for the generating function $C(u) \in \mathrm{Z}(\mathfrak{g})(u)$. This function was defined by (4.8) for $\mathfrak{g}=\mathfrak{s o}_{N}$ and by (5.10) for $\mathfrak{g}=\mathfrak{s p}_{N}$. We will use the notation and some results of Section 3. In that section we introduced in particular the rational function of $u$ valued in the algebra $\operatorname{End}\left(\mathbb{C}^{N}\right)^{\otimes N} \otimes \mathrm{U}(\mathfrak{g})$

$$
F_{\left(1^{N}\right)}(u)=\left(A_{N} \otimes 1\right) \cdot \prod_{q=1}^{N}\left(\left(1+\frac{Q_{1 q}+\ldots+Q_{q-1, q}}{2 u-q+1}\right) \otimes 1 \cdot F_{q}(u-q+1)\right)
$$

where the factors corresponding to the indices $q=1, \ldots, N$ are arranged from left to right. Recall that the element $F(u) \in \operatorname{End}\left(\mathbb{C}^{N}\right) \otimes \mathrm{U}(\mathfrak{g})(u)$ is given by $(3.2)$. 
Put $\varepsilon(u)=(2 u+1) /(2 u-N+1)$ for $\mathfrak{g}=\mathfrak{s p}_{N}$ and $\varepsilon(u)=1$ for $\mathfrak{g}=\mathfrak{s o}_{N}$. By definition $F_{\left(1^{N}\right)}(u)$ is divisible by $A_{N} \otimes 1$ on the left. Due to Proposition 3.7 and to the decomposition (3.11) the element $F_{\left(1^{N}\right)}(u)$ is also divisible by $A_{N} \otimes 1$ on the right. To show this one should use the relation (3.6) repeatedly. But the projector $A_{N}$ is one-dimensional. Therefore

$$
F_{\left(1^{N}\right)}(u)=\varepsilon(u) \cdot A_{N} \otimes \bar{C}(u)
$$

for certain rational function $\bar{C}(u)$ valued in $\mathrm{U}(\mathfrak{g})$. Moreover, due to Proposition 3.4 we have $\bar{C}(u) \in \mathrm{Z}(\mathfrak{g})(u)$. Note that the definition of $F_{\left(1^{N}\right)}(u)$ yields an explicit formula for $\bar{C}(u)$. The function $\bar{C}(u)$ is called the quantum determinant of the element $F(u)$, or the Sklyanin determinant; see $[\mathrm{O}]$ and [MNO, Section 4]. The reason for incorporating the factor $\varepsilon(u) \in \mathbb{C}(u)$ to the definition (6.1) of $\bar{C}(u)$ will become clear in the proof of Theorem 6.2.

That proof will be based on the following proposition. Consider the polynomial in $u$ valued in the algebra $\operatorname{End}\left(\mathbb{C}^{N}\right)^{\otimes N} \otimes \mathrm{U}\left(\mathfrak{g l}_{N}\right)$

$$
\left(A_{N} \otimes 1\right) \cdot E_{1}(u) E_{2}(u-1) \ldots E_{N}(u-N+1) .
$$

Using the decomposition (3.11) and the relation (3.16) the product (6.2) can be rewritten as

$$
E_{N}(u-N+1) \ldots E_{2}(u-1) E_{1}(u) \cdot\left(A_{N} \otimes 1\right) .
$$

Therefore (6.2) equals $A_{N} \otimes H(u)$ for a certain polynomial $H(u)$ valued in $\mathrm{U}\left(\mathfrak{g l}_{N}\right)$. This polynomial is called the quantum determinant of the element $E(u)$. For comments see [MNO, Section 2] and references therein. Arguments similar to those used in the proof of Proposition 3.4 show that actually $H(u) \in \mathrm{Z}\left(\mathfrak{g l}_{N}\right)(u)$. Further, similarly to the proof of Corollary 3.11 one demonstrates that

$$
\left(A_{N} \otimes 1\right) \cdot \tilde{E}_{1}(u-N+1) \ldots \tilde{E}_{N-1}(u-1) \tilde{E}_{N}(u)=A_{N} \otimes H(u) .
$$

Let $\nu$ be any partition with $\ell(\nu) \leqslant N$. Consider the corresponding irreducible $\mathfrak{g l}_{N}$-module $U_{\nu}$. The following proposition is well known, see for instance $[\mathrm{H}]$.

Proposition 6.1. The eigenvalue of $H(u)$ in the module $U_{\nu}$ is

$$
\prod_{q=1}^{N}\left(\nu_{q}+N-q-u\right) .
$$

Proof. Let us fix the Borel subalgebra in the Lie algebra $\mathfrak{g l}_{N}$ generated by the elements $E_{i j}$ with $i \leqslant j$. Further, fix the basis $\left(E_{-n,-n}, \ldots, E_{n n}\right)$ in the Cartan subalgebra of $\mathfrak{g l}_{N}$. Then the module $U_{\nu}$ is of the highest weight $\left(\nu_{1}, \ldots, \nu_{N}\right)$. Let $\xi \in U_{\nu}$ be a highest weight vector. By considering the action of the element (6.2) on the vector

$$
e_{n} \otimes \ldots \otimes e_{-n} \otimes \xi \in \operatorname{End}\left(\mathbb{C}^{N}\right)^{\otimes N} \otimes U_{\nu}
$$

and by using the definition of $H(u) \in \mathrm{Z}\left(\mathfrak{g l}_{N}\right)(u)$ we get the required statement

The following theorem is the main result of the present section; cf. [M1] and [NO]. 
Theorem 6.2. We have an equality in the algebra $\mathrm{Z}(\mathfrak{g})(u)$

$$
C(u)=\bar{C}\left(u+\frac{N}{2}-\frac{1}{2}\right) \cdot \prod_{q=1}^{N}\left(\frac{N}{2}+\frac{1}{2}-q-u-\eta\right)^{-1} .
$$

Proof. Let $V_{\lambda}$ be an irreducible $\mathfrak{g}$-module of highest weight $\lambda=\left(\lambda_{1}, \ldots, \lambda_{n}\right)$. As in Section 2 we assume that $\lambda_{n} \geqslant 0$ so that $\lambda$ is a partition with $\ell(\lambda) \leqslant n$. Due to $(2.8)$ and $(2.13)$ the eigenvalue of the element $C(u) \in \mathrm{Z}(\mathfrak{g})(u)$ in $V_{\lambda}$ equals

$$
\prod_{p=1}^{n} \frac{u^{2}-\left(\lambda_{p}+\rho_{p}\right)^{2}}{u^{2}-\rho_{p}^{2}}
$$

We have to show that the element of $\mathrm{Z}(\mathfrak{g})(u)$ at the right hand side of (6.4) has in $V_{\lambda}$ the same eigenvalue (6.5). As well as in Section 3, fix any embedding of the $\mathfrak{g l}_{N}$-module $U_{\lambda}$ to $\left(\mathbb{C}^{N}\right)^{\otimes l}$ where $l=|\lambda|$. Let $V \subset\left(\mathbb{C}^{N}\right)^{\otimes l}$ be the subspace of traceless tensors. Recall that $U_{\lambda} \cap V=V_{\lambda}$ unless $\mathfrak{g}=\mathfrak{s o}_{2 n}$ and $\lambda_{n} \neq 0$, in the latter case $U_{\lambda} \cap V=V_{\lambda} \oplus V_{\lambda^{*}}$. As well as in Section 3, by using Lemma 3.8 we can show that the restriction of the action of $F_{\left(1^{N}\right)}(u) \in \operatorname{End}\left(\mathbb{C}^{N}\right)^{\otimes N} \otimes \mathrm{U}(\mathfrak{g})(u)$ to $\left(\mathbb{C}^{N}\right)^{\otimes l} \otimes V$ coincides with that of the element from $\operatorname{End}\left(\mathbb{C}^{N}\right)^{\otimes N} \otimes \mathrm{U}\left(\mathfrak{g l}_{N}\right)(u)$

$$
\begin{gathered}
\left(A_{N} \otimes 1\right) \cdot \prod_{q=1}^{2 k} \tilde{E}_{q}(\eta-u+q-1) \times \\
\prod_{q=1}^{N}\left(\left(1+\frac{Q_{1 q}+\ldots+Q_{q-1, q}}{2 u-q+1}\right) \otimes 1\right) \cdot \prod_{q=1}^{N} \frac{1}{u-q+1-\eta} \times \\
\left(A_{N} \otimes 1\right) \cdot \prod_{q=1}^{N} E_{q}(\eta+u-q+1)
\end{gathered}
$$

where the non-commuting factors corresponding to the indices $q=1, \ldots, N$ are as usual arranged from left to right. But the element (6.6) equals

$$
\left(A_{N} \cdot \prod_{q=1}^{N}\left(1+\frac{Q_{1 q}+\ldots+Q_{q-1, q}}{2 u-q+1}\right)\right) \otimes \frac{H(\eta-u+N-1) H(\eta+u)}{(u-\eta) \ldots(u-N+1-\eta)} ;
$$

see (6.3). Further, the first tensor factor in (6.7) equals $\varepsilon(u) \cdot A_{N}$. This can be verified by direct calculation; it has been performed in [MNO, Section 4]. So by the definition (6.1) the eigenvalue of $\bar{C}(u)$ in the $\mathfrak{g}$-module $V_{\lambda}$ coincides with the eigenvalue in the $\mathfrak{g l}_{N}$-module $U_{\lambda}$ of the second tensor factor in (6.7). Due to Proposition 6.1 the latter eigenvalue equals

$$
\prod_{p=1}^{n} \frac{\left(\lambda_{p}-\eta-p+u+1\right)\left(\lambda_{p}-\eta+N-p-u\right)}{u-\eta-p+1} \cdot \prod_{q=n+1}^{N}(N-q-\eta-u) .
$$

Since $\rho_{p}=\frac{N}{2}+\frac{1}{2}-\eta-p$ for $\mathfrak{g}=\mathfrak{s o}_{2 n}, \mathfrak{s o}_{2 n+1}, \mathfrak{s p}_{2 n}$ we obtain that $\bar{C}\left(u+\frac{N}{2}-\frac{1}{2}\right)$ takes in $V_{\lambda}$ the eigenvalue

$$
\prod_{p=1}^{n} \frac{u^{2}-\left(\lambda_{p}+\rho_{p}\right)^{2}}{u^{2}-\rho_{p}^{2}} \cdot \prod_{q=1}^{N}(N-q-\eta-u)
$$


This theorem was obtained by the second author in 1991. Another proof of this theorem was given by the first author in [M1]. It uses a certain map $S_{N} \rightarrow S_{N-1}$ and provides one more formula for the generating function $C(u) \in \mathrm{Z}(\mathfrak{g})(u)$.

\section{References}

[BL] L. Biedenharn and J. Louck, A new class of symmetric polynomials defined in terms of tableaux, Advances in Appl. Math. 10 (1989), 396-438.

[C] A. Capelli, Sur les opérations dans la théorie des formes algébriques, Math. Ann. 37 (1890), 1-37.

[C1] I. Cherednik, Factorized particles on the half-line and root systems, Theor. Math. Phys. 61 (1984), 977-983.

[C2] I. Cherednik, On special bases of irreducible finite-dimensional representations of the degenerate affine Hecke algebra, Funct. Anal. Appl. 20 (1986), 87-89.

[D] J. Dixmier, Algèbres Enveloppantes, Gauthier-Villars, Paris, 1974.

[EHW] T. Enright, R. Howe and N. Wallach, A classification of unitary highest weight modules, in "Representation Theory of Reductive Groups" (P. C. Trombi, Ed.), Progress in Math. 40, Birkhäuser, Boston, 1983, pp. 97-143.

[H] R. Howe, Remarks on classical invariant theory, Trans. Amer. Math. Soc. 313 (1989), 539-570.

[HU] R. Howe and T. Umeda, The Capelli identity, the double commutant theorem, and multiplicity-free actions, Math. Ann. 290 (1991), 569-619.

[K] G. Kuperberg, Symmetries of plane partitions and the permanent-determinant method, J. Comb. Theory A 68 (1994), 115-151.

[K1] F. Knop, A Harish-Chandra homomorphism for reductive group actions, Ann. Math. 140 (1994), 253-288.

[K2] F. Knop, Symmetric and non-symmetric quantum Capelli polynomials, Comment. Math. Helvet. 72 (1997), 84-100.

[KS1] B. Kostant and S. Sahi, The Capelli identity, tube domains and the generalized Laplace transform, Adv. Math. 87 (1991), 71-92.

[KS2] B. Kostant and S. Sahi, Jordan algebras and Capelli identities, Invent. Math. 112 (1993), 657-664.

[KV] M. Kashiwara and M. Vergne, On the Segal-Shale-Weil representations and harmonic polynomials, Invent. Math. 41 (1978), 1-47.

[M] I. Macdonald, Symmetric Functions and Hall Polynomials, Clarendon Press, Oxford, 1995.

[M1] A. Molev, Sklyanin determinant, Laplace operators, and characteristic identities for classical Lie algebras, J. Math. Phys. 36 (1995), 923-943.

[M2] A. Molev, Factorial supersymmetric Schur functions and super Capelli identities, in "Kirillov's Seminar on Representation Theory" (G. I. Olshanski, Ed.), Amer. Math. Soc. Transl. 181, AMS, Providence, 1998, pp. 109-137.

[MNO] A. Molev, M. Nazarov and G. Olshanski, Yangians and classical Lie algebras, Russian Math. Surveys 51 (1996), 205-282.

[N1] M. Nazarov, Quantum Berezinian and the classical Capelli identity, Lett. Math. Phys. 21 (1991), 123-131.

[N2] M. Nazarov, Yangians and Capelli identities, in "Kirillov's Seminar on Representation Theory" (G. I. Olshanski, Ed.), Amer. Math. Soc. Transl. 181, AMS, Providence, 1998, pp. 139-164. 
[NO] M. Nazarov and G. Olshanski, Bethe subalgebras in twisted Yangians, Commun. Math. Phys. 178 (1996), 483-506.

[NUW1] M. Noumi, T. Umeda and M. Wakayama, A quantum analogue of the Capelli identity and an elementary differential calculus on $G L_{q}(n)$, Duke. Math. J. 76 (1994), 567-594.

[NUW2] M. Noumi, T. Umeda and M. Wakayama, Dual pairs, spherical harmonics and a Capelli identity in quantum group theory, Compositio Math. 104 (1996), $227-277$.

[O] G. Olshanski, Twisted Yangians and infinite-dimensional classical Lie algebras, in "Quantum Groups" (P. P. Kulish, Ed.), Lecture Notes in Math. 1510, Springer, Berlin-Heidelberg, 1992, pp. 103-120.

[O1] A. Okounkov, Quantum immanants and higher Capelli identities, Transformation Groups 1 (1996), 99-126.

[O2] A. Okounkov, Young basis, Wick formula, and higher Capelli identities, Int. Math. Research Notes (1996), 817-839.

[OO1] A. Okounkov and G. Olshanski, Shifted Schur functions, St. Petersburg Math. J. 9 (1998), 239-300.

[OO2] A. Okounkov and G. Olshanski, Shifted Schur functions II. Binomial formula for characters of classical groups and applications, in "Kirillov's Seminar on Representation Theory" (G. I. Olshanski, Ed.), Amer. Math. Soc. Transl. 181, AMS, Providence, 1998, pp. 245-271.

[S] S. Sahi, The spectrum of certain invariant differential operators associated to a Hermitian symmetric space, in "Lie Theory and Geometry" (J.-L. Brylinski, R. Brylinski, V. Guillemin, V. Kac, Eds.), Progress in Math. 123, Birkhäuser, Boston, 1994, pp. 569-576.

[S1] E. Sklyanin, Boundary conditions for integrable equations, Funct. Anal. Appl. 21 (1987), 164-166.

[S2] E. Sklyanin, Boundary conditions for integrable quantum systems, J. Phys. A 21 (1988) 2375-2389.

[W] H. Weyl, Classical Groups, their Invariants and Representations, Princeton University Press, Princeton, 1946. 